TOPOLOGICAL CRITERION FOR SELECTION OF QUINTIC PYTHAGOREAN-HODOGRAPH HERMITE INTERPOLANTS

By

Hyeong In Choi

Rida T. Farouki

Song-Hwa Kwon

and

Hwan Pyo Moon

IMA Preprint Series \# 2131

( August 2006)

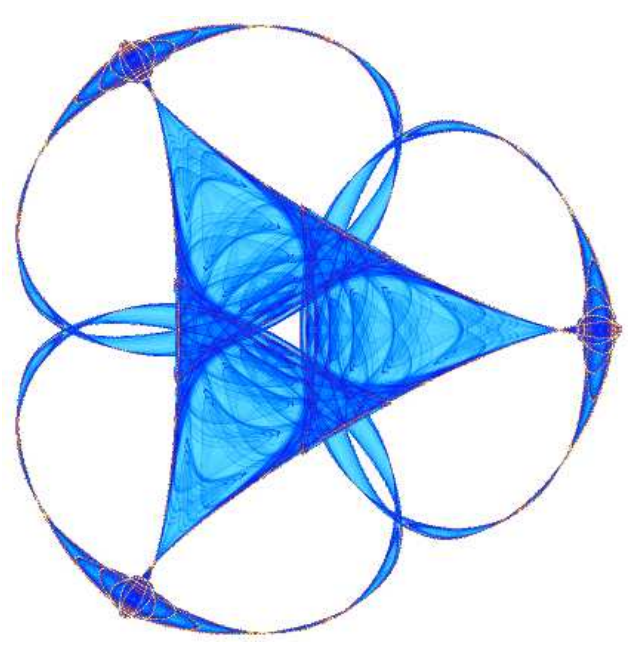

INSTITUTE FOR MATHEMATICS AND ITS APPLICATIONS

UNIVERSITY OF MINNESOTA 400 Lind Hall

207 Church Street S.E.

Minneapolis, Minnesota 55455-0436

Phone: 612-624-6066 Fax: 612-626-7370

URL: http://www.ima.umn.edu 


\title{
Topological Criterion for Selection of Quintic Pythagorean-Hodograph Hermite Interpolants*
}

\author{
Hyeong In Choi ${ }^{a}$, Rida T. Farouki ${ }^{b}$, Song-Hwa Kwon ${ }^{c, \dagger}$, Hwan Pyo Moon ${ }^{a}$ \\ ${ }^{a}$ Department of Mathematics, Seoul National University, Seoul, KOREA \\ ${ }^{b}$ Department of Mechanical and Aeronautical Engineering, \\ University of California, Davis, CA 95616, USA \\ ${ }^{c}$ Institute for Mathematics and its Application, \\ University of Minnesota, Minneapolis, MN 55455, USA
}

\begin{abstract}
A topological approach to identifying the "good" interpolant among the four distinct solutions to the first-order Hermite interpolation problem for planar quintic Pythagorean-hodograph curves is presented. An existence theorem is proved, together with a complete analysis of uniqueness/nonuniqueness properties. A simple formula for finding the "good" solution, without appealing to curve fairness or energy integrals, is also presented.
\end{abstract}

\section{Introduction}

By virtue of their special algebraic structures, the Pythagorean-hodograph (PH) curves exhibit significant computational advantages over "ordinary" polynomial parametric curves for computer-aided design and manufacturing (CAD/CAM), robotics, motion control, animation, and related applications. For example, the arc length of a $\mathrm{PH}$ curve can be computed precisely by evaluating a polynomial, and the offset curves at any fixed distance from a $\mathrm{PH}$ curve - employed as tool paths in numerical-control ( $\mathrm{NC}$ ) machining - are rational curves that can be exactly represented in CAD systems 8, 7. Digital motion control along curved paths, with fixed or variable feedrates (speeds), is a promising new application context for PH curves [4, 5, 11. The interpolation integral, which defines timed "reference points" on a curved path in accordance with the specified feedrates, admits an analytic reduction for PH curves, yielding real-time interpolators that are remarkably accurate, efficient, and versatile compared to the customary practice of approximating curved paths by linear/circular "G code" segments. Also, in the interpolation of discrete data, $\mathrm{PH}$ curves produce "fairer" loci (with

*First author also holds joint appointment in the Research Institute of Mathematics, Seoul National University. The third author is supported by Post Doctoral Fellowship from IMA.

†Corresponding author. Email address: shkwon@ima.umn.edu 
more even curvature distributions) than ordinary polynomial curves, and they admit exact evaluation of the bending energy integral $[2,10,6$. The theory and applications of $\mathrm{PH}$ curves have been extended in many directions. For example, rational $\mathrm{PH}$ curves have offsets of lower degree than polynomial $\mathrm{PH}$ curves, but are not as well-suited to motion control since their arc lengths do not depend rationally on the curve parameter [1, 16, 17. $\mathrm{PH}$ curves have also been defined in the Minkowski metric of special relativity theory, allowing exact recovery of two-dimensional shapes from their medial-axis transforms [13, 14].

Efficient and reliable methods for constructing, analyzing, and manipulating $\mathrm{PH}$ curves are essential to their practical use in CAD/CAM and other contexts. Although the formulation of such algorithms is more challenging than in the linear realm of "ordinary" Bézier/B-spline forms, substantial progress has been made. For planar PH curves, the complex-variable model [9] greatly facilitates the formulation and implementation of such algorithms for first-order Hermite interpolation [6] and construction of $C^{2}$ splines [2]. Another approach has been used to solve the local $G^{2}$ Hermite interpolation problem [12]. A common feature of such interpolation problems is that the non-linear nature of $\mathrm{PH}$ curves yields a multiplicity of formal solutions, of which typically only one has acceptable "shape" properties. The process of explicitly constructing all the interpolants in order to identify the "good" solution can be unduly cumbersome.

The solution to the first-order Hermite interpolation problem for $\mathrm{PH}$ quintics is fundamental to their practical use. Planar $\mathrm{PH}$ quintics play a role analogous to the "ordinary" cubics in free-form design, since they provide similar shape freedoms, inflectional capability, etc. Hermite interpolation allows one-for-one replacement of the "ordinary" cubics by PH quintics — and the latter typically have better curvature profiles. Moreover, by providing a designer the ability to position the first and last two control points of a PH quintic, with the Hermite algorithm "filling in" the middle two, one obtains an intuitive, interactive tool for the construction and manipulation of planar PH quintics.

In this paper, we use the complex representation [9, 6, 15, for planar curves, in which they are specified by complex-valued functions $\gamma(t)=x(t)+\mathrm{i} y(t)$ where $x(t), y(t)$ are real-valued functions of a real parameter $t$. A polynomial curve $\gamma(t) \in \mathbb{C}[t]$ is called a planar PH curve if, for some polynomial $\sigma(t) \in \mathbb{R}[t]$, its hodograph (derivative) $\gamma^{\prime}(t)=x^{\prime}(t)+\mathrm{i} y^{\prime}(t)$ satisfies the Pythagorean condition

$$
\left|\gamma^{\prime}(t)\right|^{2}=x^{\prime}(t)^{2}+y^{\prime}(t)^{2}=\sigma(t)^{2} .
$$

The hodograph of any planar $\mathrm{PH}$ curve is generated [9] by squaring a complex polynomial. More precisely, $\gamma(t)$ is a planar $\mathrm{PH}$ curve if and only if

$$
\gamma^{\prime}(t)=w(t) \chi(t)^{2}
$$

for some complex polynomial curve $\chi(t) \in \mathbb{C}[t]$ and real polynomial $w(t) \in \mathbb{R}[t]$ (see [3] for an alternative proof). We call the hodograph $\gamma^{\prime}(t)$ primitive if $x^{\prime}(t)$ and $y^{\prime}(t)$ have no common factors. A primitive Pythagorean hodograph is obtained by setting $w(t) \equiv 1$ in (1), i.e.,

$$
\gamma^{\prime}(t)=\chi(t)^{2}
$$


for a polynomial $\chi(t)=u(t)+\mathrm{i} v(t)$ with $\operatorname{gcd}(u, v)=$ constant. Every primitive $\mathrm{PH}$ curve is thus of odd degree. In this paper, we study Hermite interpolation by $\mathrm{PH}$ quintics defined by integrating (2) with a quadratic polynomial $\chi(t) \in \mathbb{C}[t]$. This problem consists of finding a planar PH quintic $\gamma(t) \in \mathbb{C}[t]$ such that

$$
\gamma(0)=p_{0}, \quad \gamma(1)=p_{1}, \quad \gamma^{\prime}(0)=a, \quad \gamma^{\prime}(1)=b
$$

for given first-order Hermite data $p_{0}, p_{1}, a, b \in \mathbb{C}$. Such curves have the form

$$
\gamma(t)=p_{0}+\int_{0}^{t} \chi(s)^{2} \mathrm{~d} s
$$

for some quadratic complex polynomial $\chi(t)$. Setting $d=p_{1}-p_{0}$, the Hermite interpolation problem corresponds to finding a quadratic $\chi(t) \in \mathbb{C}[t]$ such that

$$
\int_{0}^{1} \chi(t)^{2} \mathrm{~d} t=d, \quad \chi(0)^{2}=a, \quad \chi(1)^{2}=b .
$$

As is well-known, the same Hermite interpolation problem is uniquely solved by "ordinary" planar cubics: there is a unique cubic Bézier curve $r(t)$ such that

$$
\int_{0}^{1} r^{\prime}(t) \mathrm{d} t=d, \quad r^{\prime}(0)=a, \quad r^{\prime}(1)=b .
$$

The hodograph of this unique solution $r(t)$ is given by

$$
r^{\prime}(t)=a(1-t)^{2}+m 2(1-t) t+b t^{2}
$$

where $m=3 d-a-b$, since

$$
\int_{0}^{1} a(1-t)^{2}+m 2(1-t) t+b t^{2} \mathrm{~d} t=\frac{1}{3}(a+m+b)=d .
$$

This correspondence is more than just superficial — we show below that there is a deep connection between the cubic Bézier solution and the $\mathrm{PH}$ quintic solutions of the first-order Hermite interpolation problem. In fact, the interplay between the solutions is a central theme of this paper.

It is known [6] that there are generally four distinct solutions to the quintic $\mathrm{PH}$ interpolation problem. Consider first the equations $\chi(0)^{2}=a$ and $\chi(1)^{2}=b$. For given $a, b \in \mathbb{C}$, these equations evidently have two solutions for each of $\chi(0)$ and $\chi(1)$. Hence, there are four possible pairs $(\chi(0), \chi(1))$. Now let $(\alpha, \beta)$ be a specific pair, i.e., $\alpha^{2}=a, \beta^{2}=b$. For this pair and some $z \in \mathbb{C}$, we may write

$$
\chi(t)=\alpha(1-t)^{2}+z 2(1-t) t+\beta t^{2} .
$$

By a simple computation, the condition $\int_{0}^{1} \chi(t)^{2} \mathrm{~d} t=d$ can then be written as

$$
\left[z+\frac{3}{4}(\alpha+\beta)\right]^{2}=\frac{5}{4}\left[6 d-\alpha^{2}-\beta^{2}+\frac{1}{4}(\alpha+\beta)^{2}\right]
$$


Since, for fixed $\alpha$ and $\beta$, equation (7) is quadratic in $z$, it has two solutions. Thus, there are altogether eight possible combinations for the values of $\alpha, \beta, z$. However, one can easily see that if $\alpha, \beta, z$ is a solution, then $-\alpha,-\beta,-z$ is also a solution, and these solutions define exactly the same curve, since ${ }^{*} \gamma^{\prime}(t)=\chi(t)^{2}$. Hence, there are only four distinct $\mathrm{PH}$ quintic Hermite interpolants - they can be generated by solving (7) using pairs $(\alpha, \beta)$ and $(\alpha,-\beta)$ for any fixed $\alpha, \beta$.
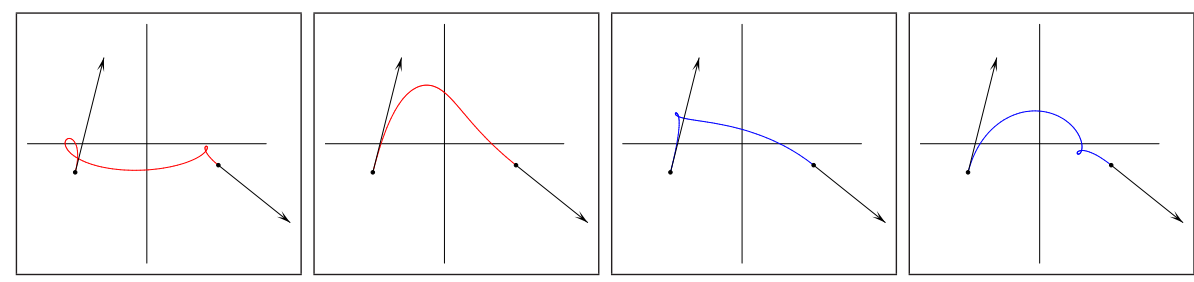

Figure 1: The four distinct $\mathrm{PH}$ quintics that interpolate given Hermite data.

Figure 1 shows an example of the four distinct PH quintic interpolants $\gamma(t)$ to given Hermite data. Only one of these four curves, the "good" solution, is a fair curve devoid of undesired loops - the other three are clearly inappropriate for practical applications. Our primary concern here is to develop methods to automatically select the "good" solution. In previous studies [10, 6] this problem was addressed by identifying the "good" solution as the one with the least value of some integral shape measure, such as the absolute rotation number $\mathcal{R}_{a b s}$ or elastic bending energy $\mathcal{E}$. The main drawback of this approach is that it can be computationally quite expensive (and different measures may sometimes suggest different choices). Another approach [15. was based on a comparison with the unique "ordinary" cubic interpolant to the specified Hermite data. It was shown that, for certain restricted Hermite data, the "good" solution could be identified a priori on the basis of the absence of anti-parallel tangents relative to the cubic.

\section{Topological perspective}

Figure 2 compares the four PH quintic interpolants in Figure 1 with the unique "ordinary" cubic interpolant to the specified Hermite data. One immediately sees that three of the four $\mathrm{PH}$ quintics differ qualitatively in shape from the cubic because of the presence of "tight loops" — in this case, it is clearly appropriate to identify the PH quintic that is free of such loops as the "good" solution. The key problem is to quantify this criterion for selecting the "good" solution in such a manner that its existence and uniqueness are guaranteed, and it can be identified by an easily-implemented and efficient algorithm. Another remarkable feature of the PH quintic interpolants is that the "looping" behavior persists under

* Clearly, there are two quadratic curves (6) from $\alpha$ to $\beta$ with middle control points $z_{1}, z_{2}$ given by the roots of (7). For brevity, we typically refer to these curves by the same symbol $\chi(t)$ but specify which of the values, $z_{1}$ or $z_{2}$, is under consideration. 
small perturbations, and even for large perturbations the loops are retained, although the solution that loops may change from one branch to another. This behavior suggests an underlying topological explanation for the phenomenon.
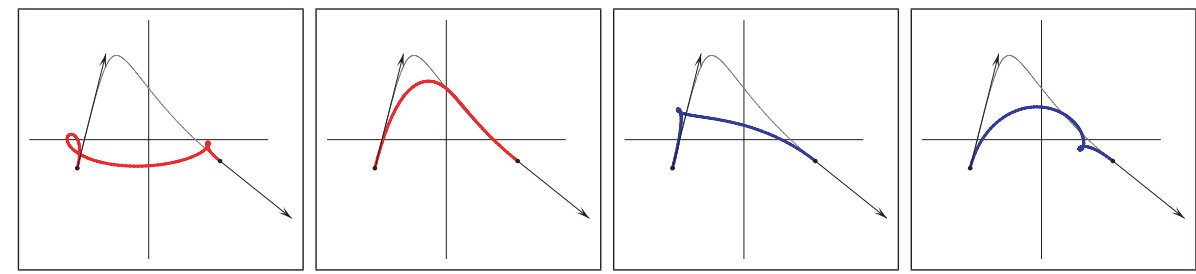

Figure 2: PH quintic interpolants compared with the unique "ordinary" cubic.

Since the "ordinary" cubic Hermite interpolant behaves reasonably well and is used extensively in practice, it is a reasonable working hypothesis that the best $\mathrm{PH}$ quintic solution should be the one that closely mimics the behavior of this cubic. According to this "principle" we would choose the second PH quintic from the left in Figure 2 as the good solution. There are many possible means to characterize similarity between the $\mathrm{PH}$ quintics and the ordinary cubic, but the most basic characterization is purely topological in nature. In this spirit we claim that, for two curves with the same boundary conditions to be "similar," we must be able to continuously deform one curve into the other (we actually deal with the curve hodographs) under fixed end points. In topological terms, this means that the winding number for the reverse concatenation (defined below) of the PH quintic and ordinary cubic hodographs should be zero. To express this criterion rigorously, we need some preliminary definitions.

Definition 2.1 Let $X(t)$ be a complex-valued continuous curve for $t \in[0,1]$. If $X(t) \neq 0$ for $t \in[0,1]$, there is a continuous real-valued function $\theta(t)$ such that

$$
X(t)=|X(t)| \operatorname{expi} \theta(t)
$$

For such an $X(t)$, the angle variation $\Delta \theta_{X}$ of $X$ is defined by

$$
\Delta \theta_{X}=\theta(1)-\theta(0) .
$$

Remark 2.2 If $X(t)$ satisfies the conditions of the above definition, so does

$$
X(t)^{2}=|X(t)|^{2} \exp \text { i } 2 \theta(t) .
$$

Since $2 \theta(t)$ is continuous, the angle variation of $X^{2}$ is given by

$$
\Delta \theta_{X^{2}}=2 \theta(1)-2 \theta(0)=2 \Delta \theta_{X} .
$$

Definition 2.3 Let $X(t)$ be a complex-valued continuous curve for $t \in[0,1]$ and suppose $X(t) \neq 0$ for all $t \in[0,1]$ and $X(0)=X(1)$. For such an $X(t)$, the winding number wind $(X)$ is defined by

$$
\text { wind }(X)=\frac{1}{2 \pi} \Delta \theta_{X}
$$


Definition 2.4 Let $X_{1}(t)$ and $X_{2}(t)$ be continuous complex-valued curves for $t \in[0,1]$ and suppose that $X_{1}(0)=X_{2}(0)$ and $X_{1}(1)=X_{2}(1)$. The reversely concatenated curve $X_{1} \ominus X_{2}$ is defined by

$$
\left(X_{1} \ominus X_{2}\right)(t)= \begin{cases}X_{1}(2 t), & t \in\left[0, \frac{1}{2}\right], \\ X_{2}(2-2 t), & t \in\left[\frac{1}{2}, 1\right] .\end{cases}
$$

Remark 2.5 Suppose $X_{1} \ominus X_{2}(t) \neq 0$ for $t \in[0,1]$. Then it is easy to see that

$$
\begin{gathered}
\Delta \theta_{X_{1} \ominus X_{2}}=\Delta \theta_{X_{1}}-\Delta \theta_{X_{2}}, \quad \Delta \theta_{X_{1} \ominus X_{2}}=-\Delta \theta_{X_{2} \ominus X_{1}}, \\
\operatorname{wind}\left(X_{1} \ominus X_{2}\right)=\frac{1}{2 \pi} \Delta \theta_{X_{1} \ominus X_{2}}=\frac{1}{2 \pi}\left(\Delta \theta_{X_{1}}-\Delta \theta_{X_{2}}\right) .
\end{gathered}
$$

We now fix some more notations and conventions. Let $a, b, d \in \mathbb{C}$ be given Hermite data. We denote the unique "ordinary" cubic interpolating the given data by $r(t)$, while $\gamma(t)$ denotes one of the four $\mathrm{PH}$ quintics that interpolate this data. We call the complex plane in which $r(t)$ and $\gamma(t)$ reside the curve space. On the other hand, the complex plane in which their hodographs $r^{\prime}(t)$ and $\gamma^{\prime}(t)$ reside is the hodograph space. We now define a key concept for this paper.

Definition 2.6 (Hodograph winding number) Since $r^{\prime}(0)=\gamma^{\prime}(0), r^{\prime}(1)=$ $\gamma^{\prime}(1)$ we can combine $r^{\prime}(t)$ and $\gamma^{\prime}(t)$ to form the reversely concatenated curve $c(t)=\left(r^{\prime} \ominus \gamma^{\prime}\right)(t)$. We call the winding number of $c=r^{\prime} \ominus \gamma^{\prime}$ with respect to the origin the hodograph winding number and denote $i i^{\prime} b y \operatorname{wind}\left(r^{\prime} \ominus \gamma^{\prime}\right)$.

For the examples in Figure 2, the reversely-concatenated hodographs $c(t)=$ $\left(r^{\prime} \ominus \gamma^{\prime}\right)(t)$ are shown in Figure 3, with their hodograph winding numbers (8).
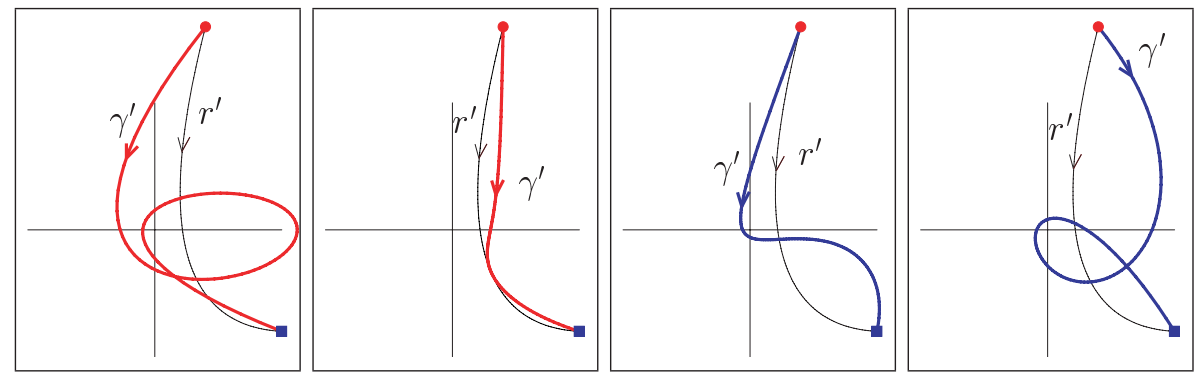

Figure 3: Reversely-concatenated hodographs $c(t)=\left(r^{\prime} \ominus \gamma^{\prime}\right)(t)$ (in hodograph space) for the ordinary cubic and PH quintics in Figure 2, From left to right, we have $\operatorname{wind}\left(r^{\prime} \ominus \gamma^{\prime}\right)=-2, \operatorname{wind}\left(r^{\prime} \ominus \gamma^{\prime}\right)=0, \operatorname{wind}\left(r^{\prime} \ominus \gamma^{\prime}\right)=-1, \operatorname{wind}\left(r^{\prime} \ominus \gamma^{\prime}\right)=1$.

The $\mathrm{PH}$ condition is characterized by the existence of a quadratic $\chi(t) \in \mathbb{C}$ satisfying (2). Since $\gamma^{\prime}(t)$ is given first and $\chi(t)$ is to be found later, this problem is identical to the path lifting problem under the branched double covering map

$$
\mathbb{C} \longrightarrow \mathbb{C}, \quad z \longmapsto z^{2} .
$$

\footnotetext{
${ }^{\dagger}$ Note that this hodograph winding number is the negative of that defined in 15.
} 
As will be seen below, this lifted path is easier to deal with than the hodograph itself. Similarly, given the hodograph $r^{\prime}(t)$ of the cubic $r(t)$, we can find a curve $s(t) \in \mathbb{C}$ satisfying the relation $r^{\prime}(t)=s(t)^{2}$. It is clear that $s(t)$ is also the lifting of $r^{\prime}(t)$ under the branched double covering map $z \longmapsto z^{2}$. Since $\gamma^{\prime}(t)$ is the square of $\chi(t)$ and $r^{\prime}(t)$ that of $s(t)$, we call $\chi(t)$ and $s(t)$ the square-root curves of $\gamma^{\prime}(t)$ and $r^{\prime}(t)$, respectively (see Figure 4). We call the complex plane in which these square-root curves reside the branched double covering space.

The three spaces employed in our arguments should be carefully noted: the curve space, the hodograph space, and the branched double covering space - for clarity, we consistently maintain them as separate copies of the complex plane.

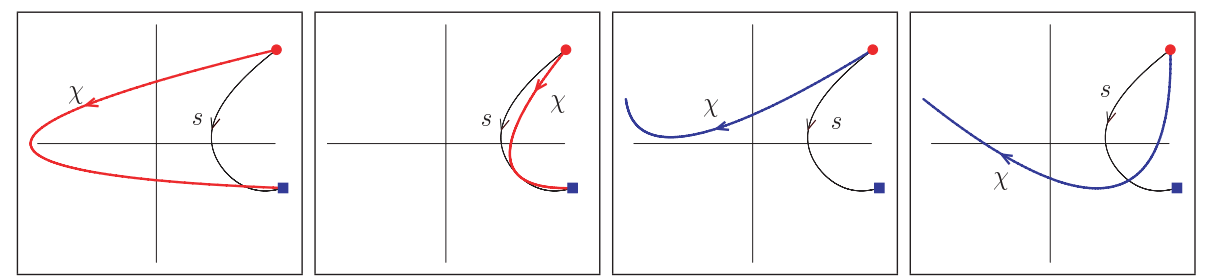

Figure 4: Hodograph square-root curves (in branched double covering space).

Now, as alluded to above, the hodograph winding number wind $\left(r^{\prime} \ominus \gamma^{\prime}\right)$ must be zero in order for a $\mathrm{PH}$ quintic to be topologically equivalent to the ordinary cubic. Thus, the following constitute the core questions addressed in this paper:

\section{Fundamental questions}

- Existence. For arbitrary Hermite data, is there at least one solution to the Hermite interpolation problem with zero hodograph winding number?

- Uniqueness. If there is such a solution, is it unique?

- Best Selection. If there exists more than one solution with zero hodograph winding number, which do we choose as the "good" solution?

The answers to the above questions are non-trivial. We first prove that the answer to the existence question is always in the affirmative. But the uniqueness question is less straightforward. For practical configurations, which predominate in applications, the answer is in the affirmative. But in special configurations, there may be two solutions with zero hodograph winding number. We clarify when such uniqueness holds or fails - in the latter case, we give a well-defined selection procedure, so the selection problem is also deemed to have been solved.

\section{$3 \quad$ Fundamental facts on quadratic curves}

We present here some basic results concerning the behavior of quadratic Bézier curves (parabola segments), which serve as the "workhorses" for our topological arguments. They appear in two different guises — as the hodographs of cubic 
Bézier curves, and as the square-root curves for the hodographs of PH quintics, and are thus critical ingredients in nearly every step of our proofs. A quadratic Bézier curve between complex numbers $z_{1}, z_{2}$ has the form

$$
Q_{z_{1}, \mu, z_{2}}(t)=z_{1}(1-t)^{2}+\mu 2(1-t) t+z_{2} t^{2}, \quad t \in[0,1]
$$

$\mu \in \mathbb{C}$ being the middle control point. If $z_{1}, z_{2}, \mu$ are understood from context, we drop the subscripts on $Q$. Similarly, we define the line segment

$$
L_{z_{1}, z_{2}}(t)=z_{1}(1-t)+z_{2} t, \quad t \in[0,1]
$$

from $z_{1}$ to $z_{2}$. If there is no danger of confusion, we drop the subscripts on $L$. For reasons that will subsequently be clear, we need detailed information on the behavior of (9) and (10). Specifically, we need to identify the conditions under which the origin lies inside the region bounded by $Q_{z_{1}, \mu, z_{2}}(t)$ and $L_{z_{1}, z_{2}}(t)$ for $t \in[0,1]$. To achieve this, we study when $Q_{z_{1}, \mu, z_{2}}(t)$ passes through the origin.

Definition 3.1 We introduce the following sets in the complex plane:

$$
\begin{aligned}
H_{z_{1}, z_{2}}:=\left\{\lambda_{1} z_{1}+\lambda_{2} z_{2} \mid \lambda_{1}<0, \lambda_{2}<0, \lambda_{1} \lambda_{2}=\frac{1}{4}\right\} \\
W_{z_{1}, z_{2}}^{0}:=\left\{\lambda_{1} z_{1}+\lambda_{2} z_{2} \mid \lambda_{1}<0, \lambda_{2}<0, \lambda_{1} \lambda_{2}<\frac{1}{4}\right\} \\
\cup\left\{\lambda_{1} z_{1}+\lambda_{2} z_{2} \mid \lambda_{1} \geq 0 \text { or } \lambda_{2} \geq 0\right\}, \\
W_{z_{1}, z_{2}}^{1}:=\left\{\lambda_{1} z_{1}+\lambda_{2} z_{2} \mid \lambda_{1}<0, \lambda_{2}<0, \lambda_{1} \lambda_{2}>\frac{1}{4}\right\}, \\
A_{z_{1}, z_{2}}:=\left\{\lambda_{1} z_{2}+\lambda_{2} z_{2} \mid \lambda_{1}>0 \text { or } \lambda_{2}>0\right\} \\
B_{z_{1}, z_{2}}:=\left\{\lambda_{1} z_{1}+\lambda_{2} z_{2} \mid \lambda_{1}<0 \text { or } \lambda_{2}<0\right\} .
\end{aligned}
$$

$H_{z_{1}, z_{2}}$ is the locus depicted in Figure [5, while $W_{z_{1}, z_{2}}^{0}, W_{z_{1}, z_{2}}^{1}, A_{z_{1}, z_{2}}, B_{z_{1}, z_{2}}$ are the shaded regions shown in Figure 6. The following theorem identifies when $Q_{z_{1}, \mu, z_{2}}$ passes through the origin.

Theorem $3.2 Q_{z_{1}, \mu, z_{2}}(t)=0$ for some $t \in(0,1)$ if and only if $\mu$ has the form $\lambda_{1} z_{1}+\lambda_{2} z_{2}$ for negative $\lambda_{1}, \lambda_{2}$ with $\lambda_{1} \lambda_{2}=\frac{1}{4}-i . e ., \mu \in H_{z_{1}, z_{2}}$.

Proof. Suppose $Q_{z_{1}, \mu, z_{2}}\left(t_{*}\right)=0$ for some $t_{*} \in(0,1)$. Then, since $z_{1}\left(1-t_{*}\right)^{2}+$ $\mu 2\left(1-t_{*}\right) t_{*}+z_{2} t_{*}^{2}=0$, we have

$$
\mu=-\frac{1}{2}\left[z_{1}\left(1-t_{*}\right) / t_{*}+z_{2} t_{*} /\left(1-t_{*}\right)\right] .
$$

Set $\lambda_{1}=-\left(1-t_{*}\right) / 2 t_{*}$ and $\lambda_{2}=-t_{*} / 2\left(1-t_{*}\right)$. Then $\lambda_{1}$ and $\lambda_{2}$ are negative, and $\lambda_{1} \lambda_{2}=\frac{1}{4}$. Conversely, suppose $\mu=\lambda_{1} z_{1}+\lambda_{2} z_{2}$ for negative $\lambda_{1}, \lambda_{2}$ with $\lambda_{1} \lambda_{2}=\frac{1}{4}$. Let $t_{*}=1 /\left(1-2 \lambda_{1}\right)$. Then, we easily infer that $Q_{z_{1}, \mu, z_{2}}\left(t_{*}\right)=0$. Also, since $\lambda_{1}$ is a negative real number, $0<t_{*}<1$.

The next theorem, which is easy to verify, gives a complete answer to the question posed above about the conditions under which the origin lies inside the region enclosed by $Q_{z_{1}, \mu, z_{2}}(t)$ and $L_{z_{1}, z_{2}}(t)$ for $t \in[0,1]$ - see Figure 7 . 


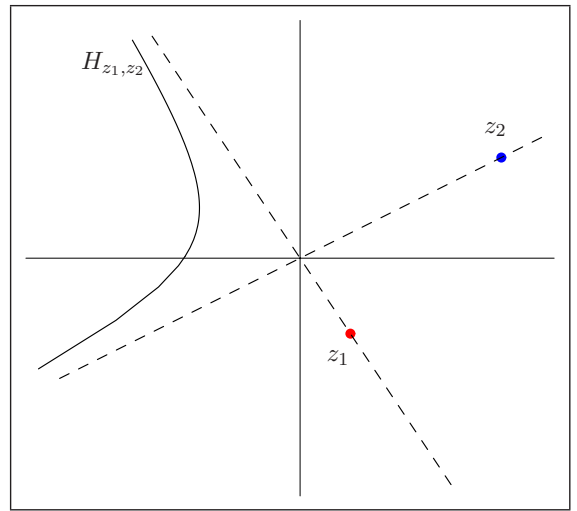

Figure 5: The locus $H_{z_{1}, z_{2}}$ is a single branch of a hyperbola.
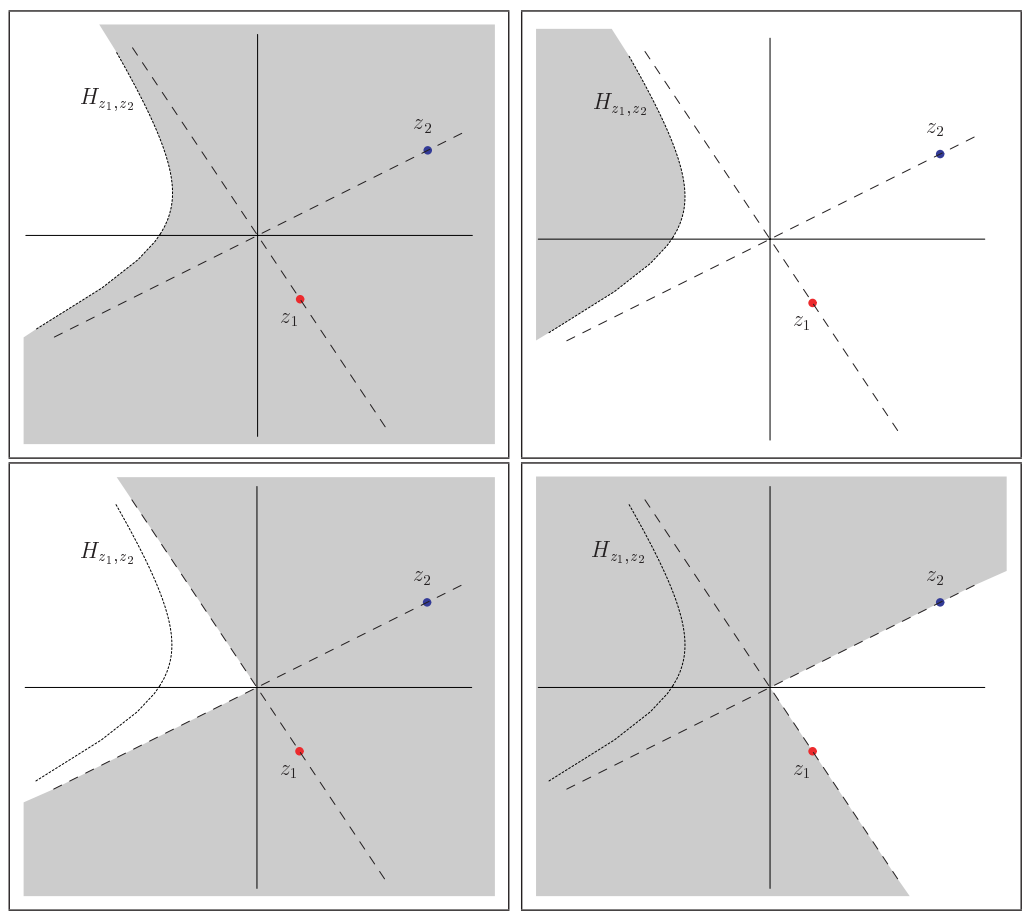

Figure 6: The four regions in the complex plane specified by Definition $3.1-$ $W_{z_{1}, z_{2}}^{0}$ (top left); $W_{z_{1}, z_{2}}^{1}$ (top right); $A_{z_{1}, z_{2}}$ (bottom left); $B_{z_{1}, z_{2}}$ (bottom right). 


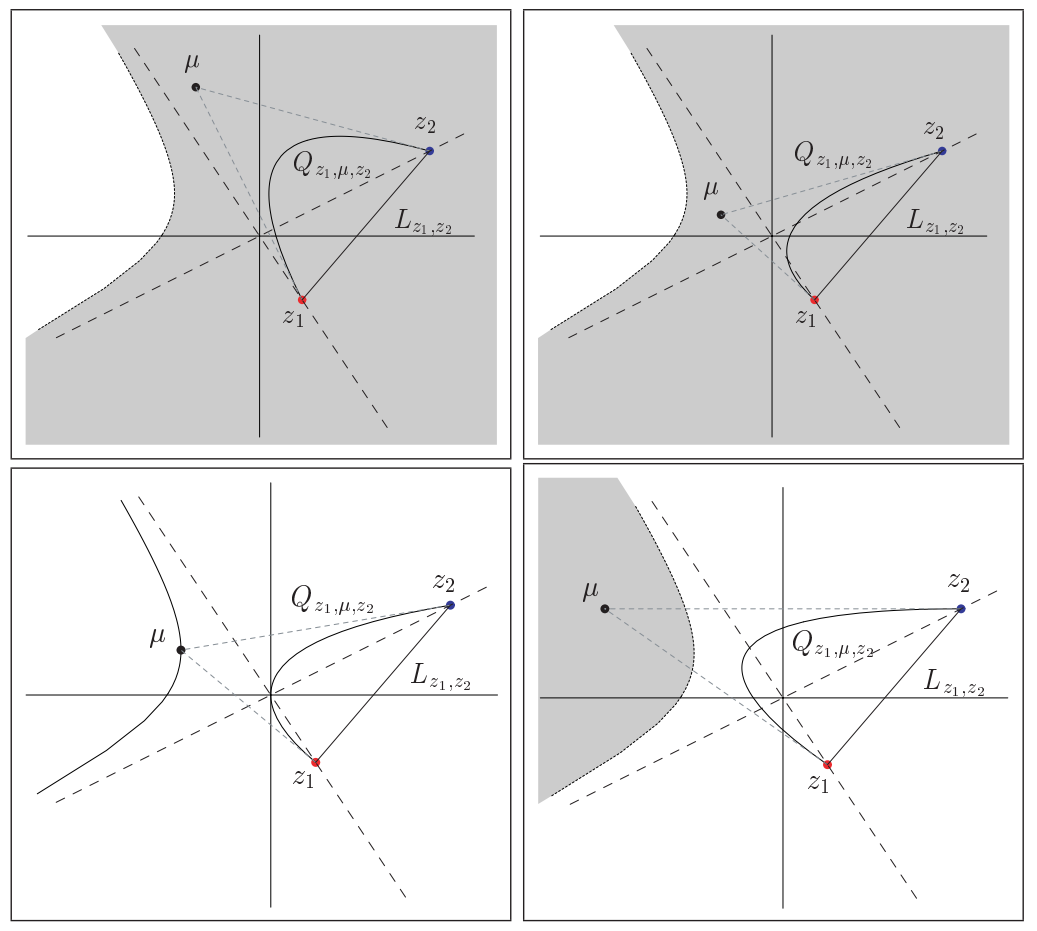

Figure 7: Illustration of Theorem 3.3 - top left: $\operatorname{wind}\left(Q_{z_{1}, \mu, z_{2}} \ominus L_{z_{1}, z_{2}}\right)=0$; top right: $\operatorname{wind}\left(Q_{z_{1}, \mu, z_{2}} \ominus L_{z_{1}, z_{2}}\right)=0$; bottom left: $\operatorname{wind}\left(Q_{z_{1}, \mu, z_{2}} \ominus L_{z_{1}, z_{2}}\right)$ is undefined; bottom right: $\operatorname{wind}\left(Q_{z_{1}, \mu, z_{2}} \ominus L_{z_{1}, z_{2}}\right)= \pm 1$.

Theorem 3.3 Suppose $z_{1}$ and $z_{2}$ are linearly independent over $\mathbb{R}$. Then, clearly $H_{z_{1}, z_{2}}, W_{z_{1}, z_{2}}^{0}, W_{z_{1}, z_{2}}^{1}$ are mutually disjoint, and $\mathbb{C}=H_{z_{1}, z_{2}} \cup W_{z_{1}, z_{2}}^{0} \cup W_{z_{1}, z_{2}}^{1}$. Also, $0 \notin A_{z_{1}, z_{2}}$ and $0 \notin B_{z_{1}, z_{2}}$. Moreover, we have:

$$
\begin{aligned}
\text { (i) } \quad \mu \in W_{z_{1}, z_{2}}^{0} & \Longleftrightarrow Q_{z_{1}, \mu, z_{2}}(t) \in A_{z_{1}, z_{2}} \text { for all } t \in(0,1) \\
& \Longleftrightarrow \operatorname{wind}\left(Q_{z_{1}, \mu, z_{2}} \ominus L_{z_{1}, z_{2}}\right)=0 \\
\text { (ii) } \quad \mu \in W_{z_{1}, z_{2}}^{1} & \Longleftrightarrow Q_{z_{1}, \mu, z_{2}}(t) \in B_{z_{1}, z_{2}} \text { for all } t \in(0,1) \\
& \Longleftrightarrow \operatorname{wind}\left(Q_{z_{1}, \mu, z_{2}} \ominus L_{z_{1}, z_{2}}\right)= \pm 1 \\
\text { (iii) } \quad \mu \in H_{z_{1}, z_{2}} & \Longleftrightarrow Q_{z_{1}, \mu, z_{2}}(t)=0 \text { for some } t \in(0,1)
\end{aligned}
$$

In case (iii), $\operatorname{wind}\left(Q_{z_{1}, \mu, z_{2}} \ominus L_{z_{1}, z_{2}}\right)$ is undefined.

The following theorem shows that the angle variation for the square-root curve $\sqrt{Q}$ of $Q$ can be simply computed from the angle variation of the line segment connecting the end points of $\sqrt{Q}$.

Theorem 3.4 Suppose $z_{1}$ and $z_{2}$ are linearly independent over $\mathbb{R}$, and let $Q=$ $Q_{z_{1}, \mu, z_{2}}(t)$. Assume further that $\mu \notin H_{z_{1}, z_{2}}$. Then the angle variation $\Delta \theta_{\sqrt{Q}}$ of 
the square-root curve $\sqrt{Q}(t)$ of $Q(t)$ for $t \in[0,1]$ is well-defined and $\Delta \theta_{\sqrt{Q}}=$ $\frac{1}{2} \Delta \theta_{Q}$. Let $L(t)$ be the line segment connecting two end points of $\sqrt{Q}(t)$ from $\sqrt{Q}(0)$ to $\sqrt{Q}(1)$. Then, the angle variation $\Delta \theta_{L}$ of $L(t)$ is well-defined, and $\Delta \theta_{\sqrt{Q}}=\Delta \theta_{L}=\operatorname{Arg}(\sqrt{Q}(1) / \sqrt{Q}(0))$, where we take $-\pi<\operatorname{Arg} z \leq+\pi$.

We also need the following lemma, whose proof is trivial.

Lemma 3.5 Let $X(t), Y(t)$ be complex-valued functions of a real parameter $t \in[0,1]$ and suppose that $X(t) \neq 0, Y(t) \neq 0$ for all $t \in[0,1]$, and $X(0)=Y(0)$. Then if $X(1)=Y(1)$, wind $\left(X^{2} \ominus Y^{2}\right)=2 \operatorname{wind}(X \ominus Y)=2 n$ for some integer $n$, and if $X(1)=-Y(1)$, wind $\left(X^{2} \ominus Y^{2}\right)=2 n+1$ for some integer $n$.

\section{Main results: generic case}

We state our key result under the following genericity condition (the remaining technicalities will be fully addressed in the next section).

Genericity assumption: For given Hermite data $a, b, d$ we assume that $a, b$ are linearly independent over $\mathbb{R}$.

Now the hodograph of the ordinary cubic $r(t)$ is a quadratic curve of the form (5), with middle control point $m$ given by

$$
m=3 d-a-b .
$$

At present, we only consider the case in which $r^{\prime}(t) \neq 0$, i.e., $m \notin H_{a, b}$ - the remaining case is dealt with in Section 4.3 .

Recall that $s(t)$ and $\chi(t)$ are the square-root curves of $r^{\prime}(t)$ and $\gamma^{\prime}(t)$, and $\alpha, \beta$ are the square roots of $a, b$. After a suitable rotation, we may assume that

$$
a=r_{1} e^{-\mathrm{i} \theta}, \quad b=r_{2} e^{\mathrm{i} \theta} .
$$

for some positive $r_{1}, r_{2}$ and $\theta \in(-\pi / 2,0) \cup(0, \pi / 2)$. We may also assume that

$$
\alpha=\sqrt{r_{1}} e^{-\mathrm{i} \theta / 2}, \quad \beta=\sqrt{r_{2}} e^{\mathrm{i} \theta / 2} .
$$

Then $\chi(t)$ is given by (6). We may also assume, without loss of generality, that $s(0)=\chi(0)=\alpha$. We then have $s(1)= \pm \beta$, and there exist two $\chi(t)$ such that $\chi(1)=\beta$ and two $\chi(t)$ such that $\chi(1)=-\beta$. We call these assumptions on $a, b$ and $\alpha, \beta$ the standard configuration, and consider them to hold henceforth. The standard-configuration locations of $a, b$ and $\alpha, \beta$ are illustrated in Figure 8 .

We now outline our answers to the fundamental questions of Section 2 by means of the hodograph winding number wind $\left(r^{\prime} \ominus \gamma^{\prime}\right)$. Our strategy is to work mostly with square-root curves $\chi(t)$ and $s(t)$ in the branched double covering space, because this greatly simplifies the problem analysis. We now present our argument through a series of reduction steps. The first reduction step concerns the square-root curve $s(t)$ of $r^{\prime}(t)$. Note that $r^{\prime}(t)$ is a quadratic curve from $a$ and $b$. Therefore, its behavior is completely determined by its middle control 

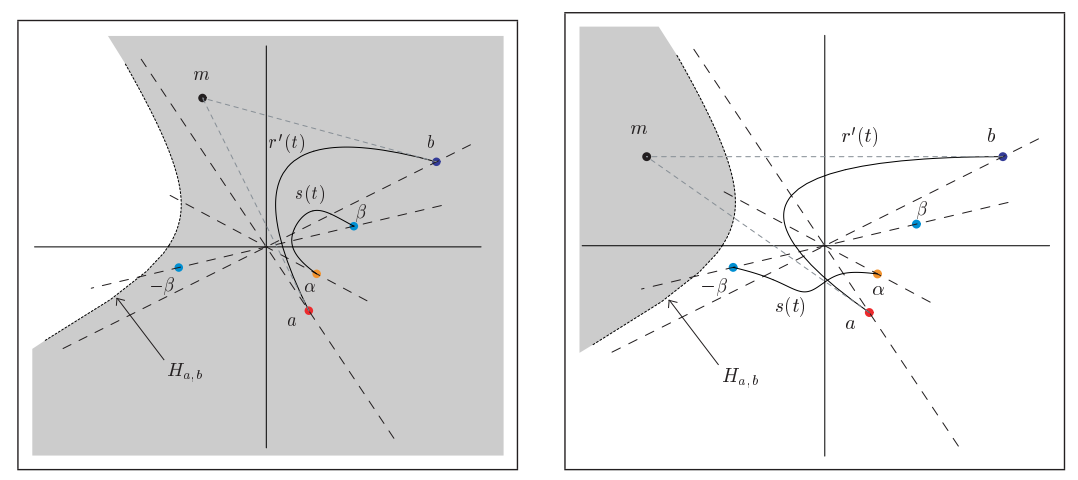

Figure 8: Locations of $a, b$ and $\alpha, \beta$ for $m \in W_{a, b}^{0}$ (left) and $m \in W_{a, b}^{1}$ (right).

point $m$ as defined in (11), which again largely governs the angle variation of $r^{\prime}(t)$. Figure 8 depicts the two cases $m \in W_{a, b}^{0}$ and $m \in W_{a, b}^{1}$. In the former case, the absolute value of the angle variation of $r^{\prime}(t)$ is less than $\pi$; in the latter, greater than $\pi$ but less than $2 \pi$. The square-root curve $s(t)$ of $r^{\prime}(t)$ is also drawn in the same complex plane as in Figure 8. By our convention, $s(0)=\alpha$ and $s(1)= \pm \beta$. Since the angle difference between $\alpha$ and $\beta$ is less than $\pi / 2, s(1)$ must be $\beta$, in the case when $m \in W_{a, b}^{0}$. Similarly, the angle difference between $\alpha$ and $-\beta$ is greater than $\pi / 2$ but less than $\pi, s(1)$ must be $-\beta$, in the case $m \in W_{a, b}^{1}$. These results are summarized in the following lemma.

Lemma 4.1 (Linear Reduction Lemma) Assume $a, b, \alpha, \beta$ are in standard configuration under the genericity assumption. Assume also that $s(0)=\alpha$. Then either one of the following holds.

Case 1: If $m \in W_{a, b}^{0}$, then $s(1)=\beta$ and the angle variation of $s(t)$ is the same as that of $L_{\alpha, \beta}(t)$, where $L_{\alpha, \beta}(t)$ is the line segment from $\alpha$ to $\beta$.

Case 2: If $m \in W_{a, b}^{1}$, then $s(1)=-\beta$ and the angle variation of $s(t)$ is the same as that of $L_{\alpha,-\beta}(t)$, where $L_{\alpha,-\beta}(t)$ is the line segment from $\alpha$ to $-\beta$.

By virtue of this lemma we can pretend, as far as the hodograph winding numbers are concerned, that the square-root curve $s(t)$ is a line segment. Hence, the problem is reduced first to enumerating all possible configurations of the quadratic curve $\chi(t)$ from $\alpha$ to $\pm \beta$ and the line segments also from $\alpha$ to $\pm \beta$, and then deciding which of these theoretical possibilities actually do or do not occur. Figure 9 illustrates all such possibilities. For example, the top left and top right cases depict the quadratic curve $\chi(t)$ from $\alpha$ to $\beta$, together with the line segments $L_{\alpha, \beta}(t)$ and $L_{\alpha,-\beta}(t)$. Note that by Lemma $4.1 L_{\alpha, \beta}(t)$ is the proxy of $s(t)$ when $s(1)=\beta$; and $L_{\alpha,-\beta}(t)$ is the proxy of $s(t)$ when $s(1)=-\beta$. The difference between these two cases is that in the former case the middle control point $z$ of $\chi(t)$ is in the region $W_{\alpha, \beta}^{0}$, thereby making the absolute value of the angle variation of $\chi(t)$ less than $\pi$, whereas in the latter case the middle 


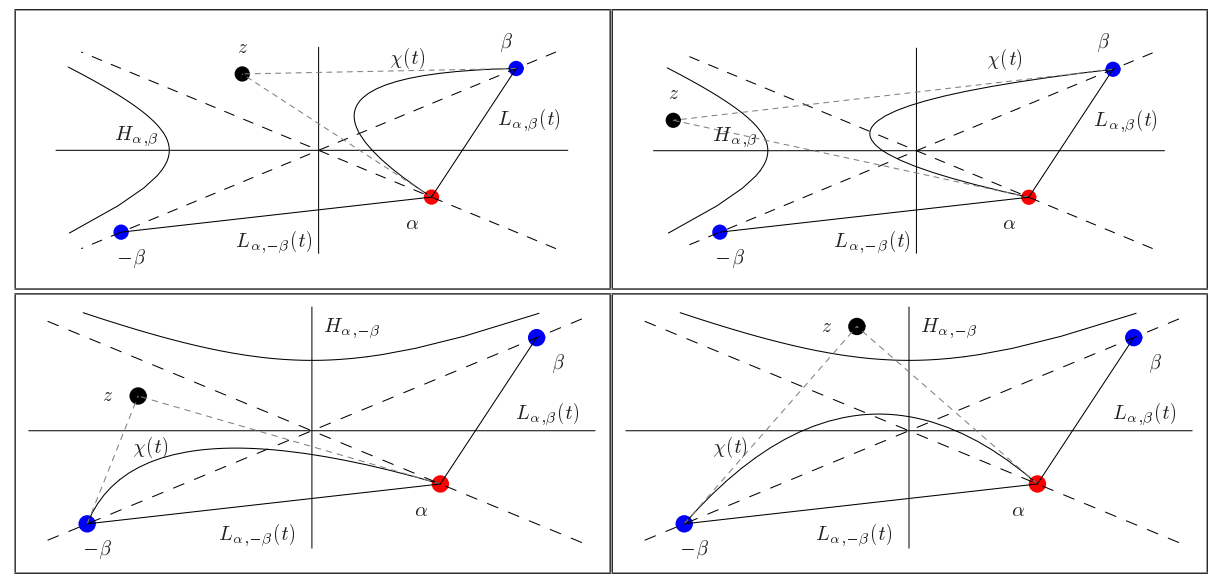

Figure 9: Possible configurations of $s(t)$ and $\chi(t)$. Top left: $\Delta \theta_{L_{\alpha, \beta}}-\Delta \theta_{\chi}=0$ and $\Delta \theta_{L_{\alpha,-\beta}}-\Delta \theta_{\chi}=-\pi$. Top right: $\Delta \theta_{L_{\alpha, \beta}}-\Delta \theta_{\chi}=2 \pi$ and $\Delta \theta_{L_{\alpha,-\beta}}-\Delta \theta_{\chi}=$ $\pi$. Bottom left: $\Delta \theta_{L_{\alpha, \beta}}-\Delta \theta_{\chi}=\pi$ and $\Delta \theta_{L_{\alpha,-\beta}}-\Delta \theta_{\chi}=0$. Bottom right: $\Delta \theta_{L_{\alpha, \beta}}-\Delta \theta_{\chi}=-\pi$ and $\Delta \theta_{L_{\alpha,-\beta}}-\Delta \theta_{\chi}=-2 \pi$.

control point $z$ of $\chi(t)$ is in the region $W_{\alpha, \beta}^{1}$, which makes the absolute value of the angle variation of $\chi(t)$ greater than $\pi$.

This angle-variation information is easily translated into the hodograph winding number. In fact, it is easy to see that the hodograph winding number wind $\left(r^{\prime} \ominus \gamma^{\prime}\right)$ is given by

$$
\operatorname{wind}\left(r^{\prime} \ominus \gamma^{\prime}\right)=\left(\Delta \theta_{L_{\alpha, \pm \beta}}-\Delta \theta_{\chi}\right) / \pi,
$$

where $\pm \operatorname{sign}$ in front of $\beta$ is chosen depending on whether $s(t)$, hence the line segment, goes from $\alpha$ to $\beta$ or $-\beta$. The angle variations $\Delta \theta_{L_{\alpha, \pm \beta}}-\Delta \theta_{\chi}$ in the branched double covering space are also depicted in the upper cases of Figure 9 . The lower cases in Figure 9 represent the same situation, but the quadratic curve $\chi(t)$ now goes from $\alpha$ to $-\beta$. Thus, the corresponding hyperbola changes from $H_{\alpha, \beta}$ to $H_{\alpha,-\beta}$. As a result, the possible values the hodograph winding number can take are restricted, as summarized in the following lemma.

Lemma 4.2 Besides the genericity assumption, assume further that $r^{\prime}(t) \neq 0$ and $\gamma^{\prime}(t) \neq 0$ for all $t \in[0,1]$. If $s(1)=\chi(1)$, then

$$
\operatorname{wind}\left(r^{\prime} \ominus \gamma^{\prime}\right)=0 \text { or } \pm 2 \text {. }
$$

On the other hand, if $s(1)=-\chi(1)$, then

$$
\text { wind }\left(r^{\prime} \ominus \gamma^{\prime}\right)= \pm 1
$$

From the above two lemmas, the existence problem can be restated as follows.

(i) If $m \in W_{a, b}^{0}$, is there at least one $\chi(t)$ such that wind $(s \ominus \chi)=0$ between two $\chi(t)$ from $\alpha$ to $\beta$ ? 
(ii) If $m \in W_{a, b}^{1}$, is there at least one $\chi(t)$ such that wind $(s \ominus \chi)=0$ between two $\chi(t)$ from $\alpha$ to $-\beta$ ?

\subsection{Existence}

We now give an answer to the existence question. Obviously, the crux of the argument involves the relation between the line segment $L_{\alpha, \pm \beta}(t)$ and $\chi(t)$ whose most important properties are stated as follows.

Lemma 4.3 If $m \in W_{a, b}^{0} \cup H_{a, b}$, then at least one of the middle control points $z$ of $\chi(t)$ from $\alpha$ to $\beta$ is in $W_{\alpha, \beta}^{0}$. If $m \in W_{a, b}^{1} \cup H_{a, b}$, at least one of the middle control points $z$ of $\chi(t)$ from $\alpha$ to $-\beta$ is in $W_{\alpha,-\beta}^{0}$.

Proof. For the first statement, suppose neither of the middle control points $z$ of $\chi(t)$ from $\alpha$ to $\beta$ is in $W_{\alpha, \beta}^{0}$. Choose any branch of the square root of the right hand side of (7), which can be written as $\lambda_{1} \alpha+\lambda_{2} \beta$ for some real numbers $\lambda_{1}$ and $\lambda_{2}$, since $\alpha$ and $\beta$ are linearly independent over $\mathbb{R}$. The middle control point $z$ of $\chi(t)$ is given by

$$
z=-\frac{3}{4}(\alpha+\beta) \pm\left(\lambda_{1} \alpha+\lambda_{2} \beta\right) .
$$

Since both values are in $H_{\alpha, \beta} \cup W_{\alpha, \beta}^{1}, \lambda_{1}$ and $\lambda_{2}$ must satisfy

$$
\begin{aligned}
& \lambda_{1}-\frac{3}{4}<0, \quad \lambda_{2}-\frac{3}{4}<0, \quad\left(\lambda_{1}-\frac{3}{4}\right)\left(\lambda_{2}-\frac{3}{4}\right) \geq \frac{1}{4}, \\
& \lambda_{1}+\frac{3}{4}>0, \quad \lambda_{2}+\frac{3}{4}>0, \quad\left(\lambda_{1}+\frac{3}{4}\right)\left(\lambda_{2}+\frac{3}{4}\right) \geq \frac{1}{4} .
\end{aligned}
$$

The region defined by these inequalities is shown as the shaded area in Figure 10 .

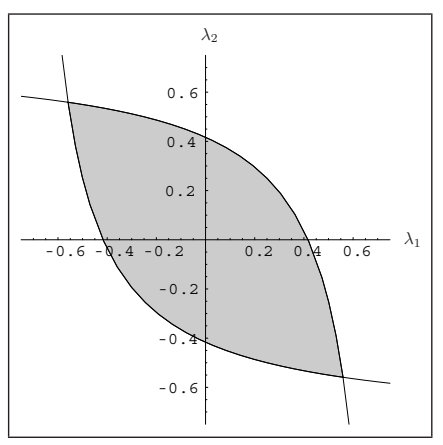

Figure 10: The region defined by the inequalities (14).

$$
\text { So, } \begin{aligned}
-\frac{\sqrt{5}}{4} \leq \lambda_{1}, \lambda_{2} \leq \frac{\sqrt{5}}{4} \text { and }-\frac{5}{16} \leq \lambda_{1} \lambda_{2} & \leq \frac{1}{16} \text {. Since } \\
\frac{5}{4}\left(6 d-a-b+\frac{1}{4}(\alpha+\beta)^{2}\right) & =\left(\lambda_{1} \alpha+\lambda_{2} \beta\right)^{2},
\end{aligned}
$$


we have

$$
\begin{aligned}
m & =3 d-a-b=\frac{2}{5}\left(\lambda_{1} \alpha+\lambda_{2} \beta\right)^{2}-\frac{1}{8}(\alpha+\beta)^{2}-\frac{1}{2}(a+b) \\
& =\left(\frac{2}{5} \lambda_{1}^{2}-\frac{5}{8}\right) a+\left(\frac{2}{5} \lambda_{2}^{2}-\frac{5}{8}\right) b+\left(\frac{4}{5} \lambda_{1} \lambda_{2}-\frac{1}{4}\right) \alpha \beta \\
& =\left(\frac{2}{5} \lambda_{1}^{2}-\frac{5}{8}+\left(\frac{4}{5} \lambda_{1} \lambda_{2}-\frac{1}{4}\right) \sqrt{r_{2} / r_{1}} / 2 \cos \theta\right) a \\
& +\left(\frac{2}{5} \lambda_{2}^{2}-\frac{5}{8}+\left(\frac{4}{5} \lambda_{1} \lambda_{2}-\frac{1}{4}\right) \sqrt{r_{1} / r_{2}} / 2 \cos \theta\right) b .
\end{aligned}
$$

Note that since

$$
\left(a / r_{1}\right)+\left(b / r_{2}\right)=2 \cos \theta
$$

$\alpha \beta$ is replaced by

$$
\alpha \beta=\frac{\sqrt{r_{1} r_{2}}}{2 \cos \theta}\left(\frac{a}{r_{1}}+\frac{b}{r_{2}}\right)
$$

in the above equation. Let $R=\sqrt{r_{2} / r_{1}}$ and

$A=\frac{2}{5} \lambda_{1}^{2}-\frac{5}{8}+\left(\frac{4}{5} \lambda_{1} \lambda_{2}-\frac{1}{4}\right) \frac{1}{2 \cos \theta} R, \quad B=\frac{2}{5} \lambda_{2}^{2}-\frac{5}{8}+\left(\frac{4}{5} \lambda_{1} \lambda_{2}-\frac{1}{4}\right) \frac{1}{2 \cos \theta} \frac{1}{R}$.

Since $\frac{2}{5} \lambda_{k}^{2}-\frac{5}{8} \leq-\frac{1}{2}(k=1,2)$ and $\frac{4}{5} \lambda_{1} \lambda_{2}-\frac{1}{4} \leq-\frac{1}{5}, A$ and $B$ are negative numbers. Moreover,

$$
A \leq-\frac{1}{2}-\frac{1}{5} \frac{1}{2 \cos \theta} R<-\frac{1}{2}, \quad B \leq-\frac{1}{2}-\frac{1}{5} \frac{1}{2 \cos \theta} \frac{1}{R}<-\frac{1}{2} .
$$

So,

$$
A B>\frac{1}{4}
$$

Since $A<0, B<0$, and $A B>\frac{1}{4}$, therefore $m \in W_{a, b}^{1}$. This is a contradiction to the assumption that $m \in W_{a, b}^{0} \cup H_{a, b}$.

For the second statement, we can similarly assume $z$ satisfies the relation

$$
z+\frac{3}{4}(\alpha-\beta)= \pm\left(\tilde{\lambda}_{1} \alpha-\tilde{\lambda}_{2} \beta\right)
$$

for some real numbers $\tilde{\lambda}_{1}$ and $\tilde{\lambda}_{2}$, where $z$ is either middle control point of $\chi(t)$. As above, we now suppose neither of the middle control points $z$ of $\chi(t)$ from $\alpha$ to $-\beta$ is in $W_{\alpha,-\beta}^{0}$. Thus, $\tilde{\lambda}_{1}$ and $\tilde{\lambda}_{2}$ satisfy the same condition (14). By a similar calculation, we get

$$
m=\tilde{A} a+\tilde{B} b
$$

where

$\tilde{A}=\frac{2}{5} \tilde{\lambda}_{1}^{2}-\frac{5}{8}-\left(\frac{4}{5} \tilde{\lambda}_{1} \tilde{\lambda}_{2}-\frac{1}{4}\right) \frac{1}{2 \cos \theta} R, \quad \tilde{B}=\frac{2}{5} \tilde{\lambda}_{2}^{2}-\frac{5}{8}-\left(\frac{4}{5} \tilde{\lambda}_{1} \tilde{\lambda}_{2}-\frac{1}{4}\right) \frac{1}{2 \cos \theta} \frac{1}{R}$.

Since $m \in W_{a, b}^{1} \cup H_{a, b}, \tilde{A}<0$ and $\tilde{B}<0$. Then, since $\frac{4}{5} \tilde{\lambda}_{1} \tilde{\lambda}_{2}-\frac{1}{4} \leq-\frac{1}{5}$,

$$
\frac{2}{5} \tilde{\lambda}_{1}^{2}-\frac{5}{8}-\left(\frac{2}{5} \tilde{\lambda}_{1} \tilde{\lambda}_{2}-\frac{1}{8}\right) R<\tilde{A}<0, \quad \frac{2}{5} \tilde{\lambda}_{2}^{2}-\frac{5}{8}-\left(\frac{2}{5} \tilde{\lambda}_{1} \tilde{\lambda}_{2}-\frac{1}{8}\right) \frac{1}{R}<\tilde{B}<0 .
$$


For ease of notation, we set

$$
M_{1}=\frac{5}{8}-\frac{2}{5} \tilde{\lambda}_{1}^{2}, \quad M_{2}=\frac{5}{8}-\frac{2}{5} \tilde{\lambda}_{2}^{2}, \quad N=\frac{1}{8}-\frac{2}{5} \tilde{\lambda}_{1} \tilde{\lambda}_{2} .
$$

With these $M_{1}, M_{2}, N$, expressions (15) are rewritten as

$$
-M_{1}+N R<\tilde{A}<0, \quad-M_{2}+N \frac{1}{R}<\tilde{B}<0 .
$$

So, $M_{1}>N R$ and $M_{2}>N / R$. By this $M_{1} M_{2}>N^{2}$. Note that $M_{1}, M_{2}, N$ are positive. Then,

$$
\begin{aligned}
\tilde{A} \tilde{B} & <M_{1} M_{2}-N\left(\frac{M_{1}}{R}+M_{2} R\right)+N^{2} \\
& \leq M_{1} M_{2}-2 N \sqrt{M_{1} M_{2}}+N^{2}=\left(\sqrt{M_{1} M_{2}}-N\right)^{2} .
\end{aligned}
$$

In addition,

$$
0<\sqrt{M_{1} M_{2}}-N \leq \frac{1}{2} M_{1}+\frac{1}{2} M_{2}-N=\frac{1}{2}-\frac{1}{5}\left(\tilde{\lambda}_{1}-\tilde{\lambda}_{2}\right)^{2} \leq \frac{1}{2} .
$$

So, $\tilde{A} \tilde{B}<\frac{1}{4}$. Since $\tilde{A}<0$ and $\tilde{B}<0, m \in W_{a, b}^{0}$. This is a contradiction to the assumption that $m \in W_{a, b}^{1} \cup H_{a, b}$.

This can be interpreted as follows. According to Lemma 4.1, the condition $m \in W_{a, b}^{0}$ implies that $s(1)=\beta$ and Lemma 4.3 says that, in this case, there is at least one $\chi(t)$ which is of the shape depicted in the top left case of Figure 9 with the corresponding line segment being $L_{\alpha, \beta}(t)$. Thus, in this case the angle variation $\Delta \theta_{L_{\alpha, \beta}}-\Delta \theta_{\chi}$ is zero, which again implies that the hodograph winding number is zero. A similar argument applies to the case when $m \in W_{a, b}^{1}$. In this case, there is at least one $\chi(t)$ such that the angle variation $\Delta \theta_{L_{\alpha, \beta}}-\Delta \theta_{\chi}$ is zero, hence the hodograph winding number is zero as in the bottom left case of Figure 9. Thus, we have the following theorem.

Theorem 4.4 (Existence Theorem) For given $p_{0}, p_{1}, a, b \in \mathbb{C}$, let $r(t)$ be the unique cubic interpolant such that

$$
r(0)=p_{0}, \quad r(1)=p_{1}, \quad r^{\prime}(0)=a, \quad r^{\prime}(1)=b .
$$

(i) If $a, b$ are linearly independent over $\mathbb{R}$ and $m=3\left(p_{1}-p_{0}\right)-a-b \notin H_{a, b}$ - i.e., $r^{\prime}(t)$ does not pass through the origin - there exists at least one quintic PH interpolant $\gamma(t)$ such that

$$
\gamma(0)=p_{0}, \quad \gamma(1)=p_{1}, \quad \gamma^{\prime}(0)=a, \quad \gamma^{\prime}(1)=b
$$

with $\operatorname{wind}\left(r^{\prime} \ominus \gamma^{\prime}\right)=0$.

(ii) $r^{\prime}(t)$ passes through the origin if and only if $m \in H_{a, b}$. In this case, $\operatorname{wind}\left(r^{\prime} \ominus \gamma^{\prime}\right)$ is undefined. 


\subsection{Uniqueness and Non-uniqueness}

Let $\chi(t)$ be the quadratic curve from $\alpha$ to either $\beta$ or $-\beta$, depending on the case. There are of course two such quadratic curves with the same start and end points, and the arguments leading to Theorem 4.4 show that at least one middle control point $z$ of $\chi(t)$ lies in the region $W_{\alpha, \beta}^{0}$ or $W_{\alpha,-\beta}^{0}$ depending on whether the middle control point $m$ of $r^{\prime}(t)$ lies in $W_{a, b}^{0}$ or $W_{a, b}^{1}$ respectively (see the convention on the use of terminology concerning how to distinguish the quadratic curves by simply designating the middle control point.)

In this section, we investigate further whether such $z$ is unique. In particular, we need to understand whether it is possible for both the $z$ points to lie in $W_{\alpha, \pm \beta}^{0}$. For that purpose, it is necessary to determine when $z$ lies at the border, i.e., on $H_{\alpha, \pm \beta}$. However, as we shall see below, it turns out that it is easier to look at the equivalent formulation in terms of the location of the middle control point $m$ of $r^{\prime}(t)$. Note that $(7)$ can be rearranged so that the middle control point $m$ of $r^{\prime}(t)$ is expressed as

$$
\begin{aligned}
m & =3 d-a-b \\
& =\left\{\begin{array}{cl}
\frac{2}{5}\left(z+\frac{3}{4}(\alpha+\beta)\right)^{2}-\frac{1}{2} \alpha^{2}-\frac{1}{2} \beta^{2}-\frac{1}{8}(\alpha+\beta)^{2} & \text { if } z \in H_{\alpha, \beta}, \\
\frac{2}{5}\left(z+\frac{3}{4}(\alpha-\beta)\right)^{2}-\frac{1}{2} \alpha^{2}-\frac{1}{2} \beta^{2}-\frac{1}{8}(\alpha-\beta)^{2} & \text { if } z \in H_{\alpha,-\beta},
\end{array}\right.
\end{aligned}
$$

which suggests the following definition.

Definition 4.5 Let $\psi_{\alpha, \beta}$ be the map from $H_{\alpha, \beta}$ to $\mathbb{C}$ defined by

$$
\psi_{\alpha, \beta}(z)=\frac{2}{5}\left(z+\frac{3}{4}(\alpha+\beta)\right)^{2}-\frac{1}{2} \alpha^{2}-\frac{1}{2} \beta^{2}-\frac{1}{8}(\alpha+\beta)^{2} .
$$

Similarly define $\psi_{\alpha,-\beta}$ as the map from $H_{\alpha,-\beta}$ to $\mathbb{C}$ defined by

$$
\psi_{\alpha,-\beta}(z)=\frac{2}{5}\left(z+\frac{3}{4}(\alpha-\beta)\right)^{2}-\frac{1}{2} \alpha^{2}-\frac{1}{2} \beta^{2}-\frac{1}{8}(\alpha-\beta)^{2} .
$$

Let $S H_{\alpha, \pm \beta}$ be the image of $H_{\alpha, \pm \beta}$ under the map $\psi_{\alpha, \pm \beta}$. In other words,

$$
\begin{aligned}
S H_{\alpha, \beta} & =\left\{\frac{2}{5}\left(z+\frac{3}{4}(\alpha+\beta)\right)^{2}-\frac{1}{2} \alpha^{2}-\frac{1}{2} \beta^{2}-\frac{1}{8}(\alpha+\beta)^{2} \mid z \in H_{\alpha, \beta}\right\}, \\
S H_{\alpha,-\beta} & =\left\{\frac{2}{5}\left(z+\frac{3}{4}(\alpha-\beta)\right)^{2}-\frac{1}{2} \alpha^{2}-\frac{1}{2} \beta^{2}-\frac{1}{8}(\alpha-\beta)^{2} \mid z \in H_{\alpha,-\beta}\right\} .
\end{aligned}
$$

Note that $S H_{\alpha, \beta}$ is a curve obtained by suitably translating and squaring the hyperbola $H_{\alpha, \beta}$ followed by another translation. From this definition, it is clear that if $m \in S H_{\alpha, \beta}$ (resp. $S H_{\alpha,-\beta}$ ), there exist at least one $z \in H_{\alpha, \beta}$ (resp. $\left.H_{\alpha,-\beta}\right)$ which is the middle control point of the quadratic curve $\chi(t)$ from $\alpha$ to $\beta$ (resp. to $-\beta$ ). In other words, if $m \in S H_{\alpha, \beta}$ (resp. $S H_{\alpha,-\beta}$ ), at least one solution $\chi(t)$ from $\alpha$ to $\beta$ (resp. $-\beta$ ) passes through the origin. In fact, the number of solutions $\chi(t)$ passing through the origin for given $m$ is $\left|\psi_{\alpha, \pm \beta}^{-1}(m)\right|$. In order to properly understand the problem, we need the following lemmas. 


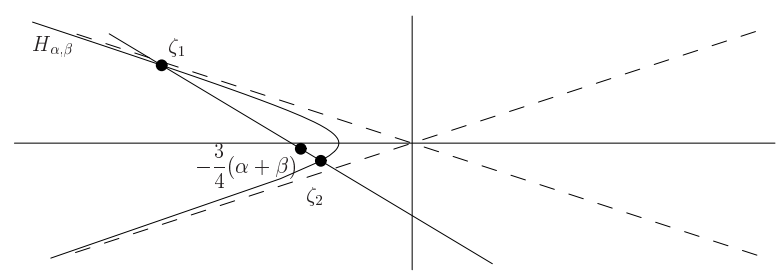

Figure 11: Proof of Lemma 4.6.

Lemma 4.6 $S H_{\alpha, \beta}$ (resp. $S H_{\alpha,-\beta}$ ) has a single self-intersection point.

Proof. The self-intersection point occurs when there are two distinct $z_{1}, z_{2} \in$ $H_{\alpha, \beta}$ such that

$$
\left(z_{1}+\frac{3}{4}(\alpha+\beta)\right)^{2}=\left(z_{2}+\frac{3}{4}(\alpha+\beta)\right)^{2} .
$$

Note that

$$
-\frac{3}{4}(\alpha+\beta)=\lambda_{1} \alpha+\lambda_{2} \beta,
$$

where $\lambda_{1}=\lambda_{2}=-\frac{3}{4}$. Therefore, at $-\frac{3}{4}(\alpha+\beta), \lambda_{1}<0, \lambda_{2}<0$, and $\lambda_{1} \lambda_{2}=$ $\frac{9}{16}>\frac{1}{4}$ so $-\frac{3}{4}(\alpha+\beta) \in W_{\alpha, \beta}^{1}$. In other words, $-\frac{3}{4}(\alpha+\beta)$ lies in the left (i.e., convex) side of $H_{\alpha, \beta}$. In Figure 11, a line through $-\frac{3}{4}(\alpha+\beta)$ is drawn. Here, $\zeta_{1}$ and $\zeta_{2}$ are defined to be the points on $H_{\alpha, \beta}$ at which this line meets with $H_{\alpha, \beta}$. In this example, the line is drawn in such a way that

$$
\left|\zeta_{1}+\frac{3}{4}(\alpha+\beta)\right|>\left|\zeta_{2}+\frac{3}{4}(\alpha+\beta)\right| .
$$

As the line rotates clockwise about the point $-\frac{3}{4}(\alpha+\beta),\left|\zeta_{1}+\frac{3}{4}(\alpha+\beta)\right|$ decreases while $\left|\zeta_{2}+\frac{3}{4}(\alpha+\beta)\right|$ increases until they become equal. As the rotation proceeds beyond this point, it is easy to see that the inequality

$$
\left|\zeta_{1}+\frac{3}{4}(\alpha+\beta)\right|<\left|\zeta_{2}+\frac{3}{4}(\alpha+\beta)\right|
$$

persists until the line becomes horizontal. In this manner, one can easily deduce the existence and uniqueness of the self-intersection point.

This lemma implies that on $S H_{\alpha, \beta}$ (resp. $S H_{\alpha,-\beta}$ ), there exists a unique $m$ such that $\left|\psi_{\alpha, \beta}^{-1}(m)\right|=2$ (resp. $\left|\psi_{\alpha,-\beta}^{-1}(m)\right|=2$ ). Thus, only at the unique self-intersection point of $S H_{\alpha, \beta}$ (resp. $S H_{\alpha,-\beta}$ ), both cases of $\chi(t)$ pass through the origin. At any other points on $S H_{\alpha, \beta}$ (resp. $S H_{\alpha,-\beta}$ ), there exists only one case of $\chi(t)$ which does not pass through the origin. At the point which is not on $S H_{\alpha, \beta}$ (resp. $S H_{\alpha,-\beta}$ ), neither case of $\chi(t)$ passes through the origin.

This lemma also means that $S H_{\alpha, \beta}$ (resp. $S H_{\alpha,-\beta}$ ) divides the complex plane $\mathbb{C}$ into three connected components (one bounded and two unbounded). Each of these connected component has a crucial influence on the winding behavior of $\chi(t)$. The following lemma, whose proof is trivial by the continuity argument, is nonetheless one of the key ingredients in our subsequent discussion.

\section{Lemma 4.7}


(i) Let $\mathcal{U}$ be an open connected component of $\mathbb{C}-S H_{\alpha, \beta}$, and let $m \in \mathcal{U}$ be the middle control point of $r^{\prime}(t)$ from a to $b$. Suppose $z_{1}, z_{2}$ are two middle control points of $\chi(t)$ from $\alpha$ to $\beta$. Then, as $m$ varies throughout $\mathcal{U}, z_{1}$ (resp. $z_{2}$ ) remains in the same set once it is in $W_{\alpha, \beta}^{0}$ or $W_{\alpha, \beta}^{1}$.

(ii) Let $\mathcal{U}$ be an open connected component of $\mathbb{C}-S H_{\alpha,-\beta}$, and let $m \in \mathcal{U}$ be the middle control point of $r^{\prime}(t)$ from a to $b$. Suppose $z_{1}, z_{2}$ are two middle control points of $\chi(t)$ from $\alpha$ to $-\beta$. Then, as $m$ varies throughout $\mathcal{U}, z_{1}$ (resp. $z_{2}$ ) remains in the same set once it is in $W_{\alpha,-\beta}^{0}$ or $W_{\alpha,-\beta}^{1}$.

For fixed $a$ and $b$, the number of $z$ in $W_{\alpha, \beta}^{0}\left(\operatorname{resp} . W_{\alpha,-\beta}^{0}\right)$ depends only on the value of $m$. Let us denote this number by $\mathcal{N}_{\alpha, \beta}(m)$, resp. $\mathcal{N}_{\alpha,-\beta}(m)$. However, from the above lemma, this number is constant on the each open connected component $\mathcal{U}$. Let us denote this number by $\mathcal{N}_{\alpha, \beta}(\mathcal{U})$, resp. $\mathcal{N}_{\alpha,-\beta}(\mathcal{U})$. From (7), we can easily deduce that the mid-point of the two middle control points $z_{1}$ and $z_{2}$ of $\chi(t)$ starting from $\alpha$ ending at $\beta$ is $-\frac{3}{4}(\alpha+\beta)$ and the distance between $z_{1}$ (or $\left.z_{2}\right)$ and $-\frac{3}{4}(\alpha+\beta)$ is the square root of the modulus of

$$
\frac{5}{4}\left(2 m+a+b+\frac{1}{4}(\alpha+\beta)^{2}\right) .
$$

Since $-\frac{3}{4}(\alpha+\beta)$ is in the left side of $H_{\alpha, \beta}$, at least one $z$ is in the right side of $H_{\alpha, \beta}$, i.e., in $W_{\alpha, \beta}^{0}$, if $|m|$ is sufficiently large. So, for the unbounded component $\mathcal{U}$, the number of $z \in W_{\alpha, \beta}^{0}$ cannot be zero. We thus have the following lemma.

Lemma 4.8 If $\mathcal{U}$ is an unbounded open connected component of $\mathbb{C}-S H_{\alpha, \beta}$ $\left(\right.$ resp. $\left.\mathbb{C}-S H_{\alpha,-\beta}\right), \mathcal{N}_{\alpha, \beta}(\mathcal{U}) \neq 0\left(\operatorname{resp} . \mathcal{N}_{\alpha,-\beta}(\mathcal{U}) \neq 0\right)$.

For $m$ which makes the value of (16) zero, $z_{1}$ and $z_{2}$ must be $-\frac{3}{4}(\alpha+\beta)$. Thus, $\mathcal{N}_{\alpha, \beta}(m)=0$ for such $m$. This implies that there exists at least one open connected component $\mathcal{U}$ such that $\mathcal{N}_{\alpha, \beta}(\mathcal{U})=0$. Thus, from the above lemma, we can get the following lemma.

Lemma 4.9 If $\mathcal{U}$ is the bounded open connected component of $\mathbb{C}-S H_{\alpha, \beta}$ (resp. $\left.\mathbb{C}-S H_{\alpha,-\beta}\right), \mathcal{N}_{\alpha, \beta}(\mathcal{U})=0\left(\operatorname{resp} . \mathcal{N}_{\alpha,-\beta}(\mathcal{U})=0\right)$.

We will call this bounded open connected component the island.

If $m$ goes infinitely to the right along a horizontal line, the argument of (16) goes to 0 . Thus, $z_{1}, z_{2}$ are on the line which passes through $-\frac{3}{4}(\alpha+\beta)$ and is almost parallel to the $x$-axis. Since the distance between $z_{1}$ (or $z_{2}$ ) and $-\frac{3}{4}(\alpha+\beta)$ becomes large enough, $\mathcal{N}_{\alpha, \beta}(m)$ becomes 1 as $m$ goes infinitely to the right along a horizontal line. By similar arguments, we obtain the following.

\section{Lemma 4.10 (Asymptotic Behavior)}

If $m$ moves to the right on a horizontal line, $\mathcal{N}_{\alpha, \beta}(m)$ eventually becomes 1.

If $m$ moves to the left on a horizontal line, $\mathcal{N}_{\alpha, \beta}(m)$ eventually becomes 2 .

If $m$ moves in any direction on a vertical line, $\mathcal{N}_{\alpha, \beta}(m)$ eventually becomes 2. 

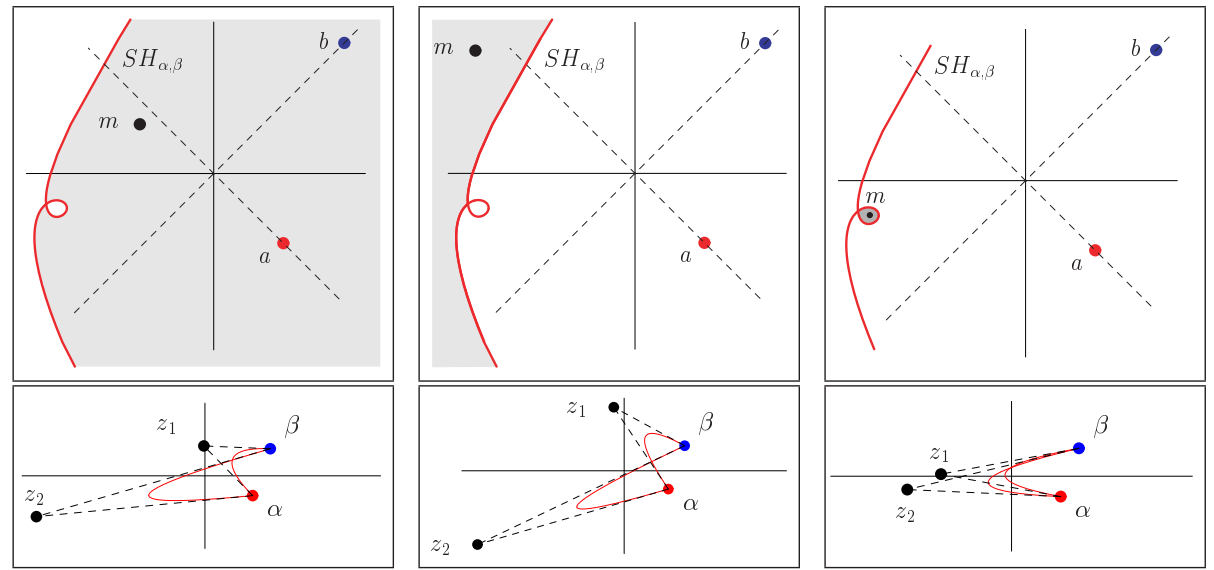

Figure 12: $S H_{\alpha, \beta}$ for $m$ lying in the different open connected components of $\mathbb{C}-S H_{\alpha, \beta}$ assuming $m \in W_{a, b}^{0}$, and thus $s(t)$ going from $\alpha$ to $\beta$. Left: the case $m \in \mathcal{N}_{\alpha, \beta}^{1}$ with $z_{1} \in W_{\alpha, \beta}^{0}$ and $z_{2} \in W_{\alpha, \beta}^{1}$. Center: the case $m \in \mathcal{N}_{\alpha, \beta}^{2}$ with $z_{1}, z_{2} \in W_{\alpha, \beta}^{0}$. Right: the case $m \in \mathcal{N}_{\alpha, \beta}^{0}$ with $z_{1}, z_{2} \in W_{\alpha, \beta}^{1}$.

If $m$ moves to the right on a horizontal line, $\mathcal{N}_{\alpha,-\beta}(m)$ eventually becomes 2 .

If $m$ moves to the left on a horizontal line, $\mathcal{N}_{\alpha,-\beta}(m)$ eventually becomes 1.

If $m$ moves in any direction on a vertical line, $\mathcal{N}_{\alpha,-\beta}(m)$ eventually becomes 1.

Since there are exactly three open connected components of $\mathbb{C}-S H_{\alpha, \beta}$ (resp. $\left.\mathbb{C}-S H_{\alpha,-\beta}\right)$, there exists only one open connected component $\mathcal{U}$ such that $\mathcal{N}_{\alpha, \beta}(\mathcal{U})=k\left(\operatorname{resp} . \mathcal{N}_{\alpha,-\beta}(\mathcal{U})=k\right)$ for each $k=0,1,2$. Let us denote the open connected component $\mathcal{U}$ of $\mathbb{C}-S H_{\alpha, \beta}$ (resp. $\left.\mathbb{C}-S H_{\alpha,-\beta}\right)$ with $\mathcal{N}_{\alpha, \beta}(\mathcal{U})=k$ $\left(\right.$ resp. $\left.\mathcal{N}_{\alpha,-\beta}(\mathcal{U})=k\right)$ by $\mathcal{N}_{\alpha, \beta}^{k}\left(\operatorname{resp} . \mathcal{N}_{\alpha,-\beta}^{k}\right)$. Then, from the above lemma, $\mathcal{N}_{\alpha, \beta}^{1}$ is in the right side of $S H_{\alpha, \beta}, \mathcal{N}_{\alpha, \beta}^{2}$ is in the left side of $S H_{\alpha, \beta}, \mathcal{N}_{\alpha,-\beta}^{1}$ is in the left side of $S H_{\alpha,-\beta}$, and $\mathcal{N}_{\alpha,-\beta}^{2}$ is in the right side of $S H_{\alpha,-\beta}$.

\section{Theorem 4.11}

(a) Among the three open connected components of $\mathbb{C}-S H_{\alpha, \beta}, \mathcal{N}_{\alpha, \beta}^{0}$ is the only bounded component, while $\mathcal{N}_{\alpha, \beta}^{1}$ and $\mathcal{N}_{\alpha, \beta}^{2}$ are the unbounded components to right and left of $S H_{\alpha, \beta}$ respectively.

(b) Among the three open connected components of $\mathbb{C}-S H_{\alpha,-\beta}, \mathcal{N}_{\alpha,-\beta}^{0}$ is the only bounded component, while $\mathcal{N}_{\alpha,-\beta}^{1}$ and $\mathcal{N}_{\alpha,-\beta}^{2}$ are the unbounded components to the left and right of $\mathrm{SH}_{\alpha,-\beta}$ respectively.

Figure 12 depicts $S H_{\alpha, \beta}$ for $m$ lying in the different open connected components of $\mathbb{C}-S H_{\alpha, \beta}$ assuming $m \in W_{a, b}^{0}$, thus $s(t)$ going from $\alpha$ to $\beta$ (note that, under this assumption, the case for $\chi(t)$ going from $\alpha$ to $-\beta$ need not be 

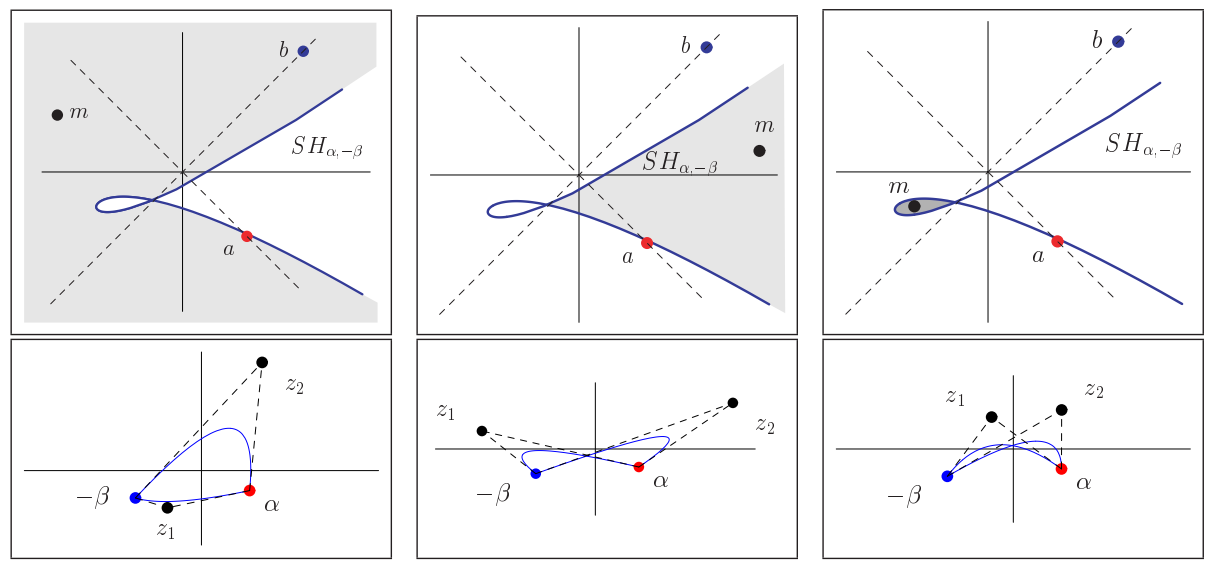

Figure 13: Analogs to the cases shown in Figure 12, when $\beta$ is replaced by $-\beta$. Left: $m \in \mathcal{N}_{\alpha,-\beta}^{1}$ with $z_{1} \in W_{\alpha,-\beta}^{0}$ and $z_{2} \in W_{\alpha,-\beta}^{1}$. Center: $m \in \mathcal{N}_{\alpha,-\beta}^{2}$ with $z_{1}, z_{2} \in W_{\alpha,-\beta}^{0}$. Right: $m \in \mathcal{N}_{\alpha,-\beta}^{0}$ with $z_{1}, z_{2} \in W_{\alpha,-\beta}^{1}$.

considered as in the case $\left.\operatorname{wind}\left(r^{\prime} \ominus \gamma^{\prime}\right)= \pm 1\right)$. For example, in the top row $m$ lies to the right of $S H_{\alpha, \beta}$, which gives rise to the situation in which one of the middle control points $z$ is in $W_{\alpha, \beta}^{0}$, while the other in $W_{\alpha, \beta}^{1}$. The middle row represents the case in which $m$ lies entirely to the left of $S H_{\alpha, \beta}$ which gives rise to the situation in which both the middle control points $z$ lies in $W_{\alpha, \beta}^{0}$. Finally, the bottom row represents the case in which $m$ lies in the small island, which incurs the situation in which both the middle control points $z$ lie in $W_{\alpha, \beta}^{1}$. Figure 13 illustrates the analogous cases in which $\beta$ is replaced by $-\beta$, with corresponding changes in the assumption that $m \in W_{a, b}^{1}$. Note that Figures 12 and 13 represent all the theoretical possibilities to be considered.

Our next step is to show that not all the six theoretical possibilities in Figure 12 and 13 actually occur, and in fact we will decide which ones actually occur and which ones don't. For this, we need the following lemmas.

Lemma 4.12 $S H_{\alpha, \beta}$ meets $H_{a, b}$ in least at two points, and the island of $S H_{\alpha, \beta}$ with its boundary is entirely in the left side of $H_{a, b}$.

Proof. When $m$ is in the island or on its boundary, then clearly both $z$ lie in $W_{\alpha, \beta}^{1} \cup H_{\alpha, \beta}$ by Lemma 4.9 and the continuity argument as depicted in Figure 12. Suppose there exists an $m$ in the island or on its boundary, such that $m$ is in the right side of $H_{a, b}$ or on $H_{a, b}$, i.e., $m \in W_{a, b}^{0} \cup H_{a, b}$, then by Lemma 4.3 there exists at least one $z \in W_{\alpha, \beta}^{0}$. But this is a contradiction. Therefore, none of the points in the island or on its boundary can be in the right side of $H_{a, b}$. In fact, we have shown that the island with its boundary is included in open set $W_{a, b}^{1}$. Thus, $H_{a, b}$ does not intersect the island, even in its boundary.

Let us now show that they meet in least at two points. Recall that $a, b, \alpha$, $\beta$ are in standard configuration, which means that we may assume they satisfy 
(12) and (13). $H_{a, b}$ has two asymptotic half lines whose arguments (i.e., angle from the positive $x$-axis) are $\pi-\frac{1}{2} \theta$ and $\pi+\frac{1}{2} \theta$ respectively. By inspection, it is trivial to see that $S H_{\alpha, \beta}$ converges asymptotically to two half lines whose arguments are $2 \pi-\theta \equiv-\theta(\bmod 2 \pi)$ and $2 \pi+\theta \equiv \theta(\bmod 2 \pi)$. Since the island of $S H_{\alpha, \beta}$ is in the left side of $H_{a, b}$, the asymptotic half line of $S H_{\alpha, \beta}$ with argument $\theta$ must meet with the asymptotic half line of $H_{a, b}$ with argument $\pi-\theta$. Therefore by continuity, the curve $S H_{\alpha, \beta}$ must meet $H_{a, b}$ in one side of the island when an orientation for the curve $S H_{\alpha, \beta}$ is fixed. Similar argument shows that $S H_{\alpha, \beta}$ must meet $H_{a, b}$ in the other side of island.

Lemma 4.13 If $m \in W_{a, b}^{1} \cup H_{a, b}$, at least one middle control point $z$ of $\chi(t)$ from $\alpha$ to $-\beta$ is in $W_{\alpha,-\beta}^{1}$.

Proof. This proof is very similar to that of the second statement in Lemma 4.3 . Let $z+\frac{3}{4}(\alpha-\beta)= \pm\left(\lambda_{1} \alpha-\lambda_{2} \beta\right)$. We suppose both of $z$ are in $H_{\alpha,-\beta} \cup W_{\alpha,-\beta}^{0}$. Then, $\lambda_{1}, \lambda_{2}$ satisfy $\left|\lambda_{1}\right|,\left|\lambda_{2}\right| \geq \frac{\sqrt{5}}{4}, \lambda_{1} \lambda_{2} \leq-\frac{5}{16}$. If we let $R=\sqrt{r_{2} / r_{1}}$, by the similar calculation, we get

$$
m=A a+B b
$$

where

$A=\frac{2}{5} \lambda_{1}^{2}-\frac{5}{8}-\left(\frac{4}{5} \lambda_{1} \lambda_{2}-\frac{1}{4}\right) \frac{1}{2 \cos \theta} R, \quad B=\frac{2}{5} \lambda_{2}^{2}-\frac{5}{8}-\left(\frac{4}{5} \lambda_{1} \lambda_{2}-\frac{1}{4}\right) \frac{1}{2 \cos \theta} \frac{1}{R}$.

Since $m \in W_{a, b}^{1} \cup H_{a, b}, A<0$ and $B<0$. For the ease of notation, set

$$
M_{1}=\frac{5}{8}-\frac{2}{5} \lambda_{1}^{2}, \quad M_{2}=\frac{5}{8}-\frac{2}{5} \lambda_{2}^{2}, \quad N=\frac{1}{8}-\frac{2}{5} \lambda_{1} \lambda_{2} .
$$

Since $N \geq \frac{1}{4}>0$, we can get inequalities

$$
\begin{aligned}
-M_{1}+N R<-M_{1}+N R / \cos \theta & =A<0, \\
-M_{2}+N / R<-M_{2}+N / R \cos \theta & =B<0 .
\end{aligned}
$$

So, $M_{1}>N R$ and $M_{2}>N / R$. By this, $M_{1} M_{2}>N^{2}$. Then,

$$
\begin{aligned}
A B & <M_{1} M_{2}-N\left(M_{1} / R+M_{2} R\right)+N^{2} \\
& \leq M_{1} M_{2}-2 N \sqrt{M_{1} M_{2}}+N^{2}=\left(\sqrt{M_{1} M_{2}}-N\right)^{2} .
\end{aligned}
$$

In addition,

$$
0<\sqrt{M_{1} M_{2}}-N \leq \frac{1}{2} M_{1}+\frac{1}{2} M_{2}-N=\frac{1}{2}-\frac{1}{5}\left(\lambda_{1}-\lambda_{2}\right)^{2} \leq \frac{1}{2} .
$$

So, $A B<\frac{1}{4}$. Since $A<0$ and $B<0, m \in W_{a, b}^{0}$. This is a contradiction to the assumption that $m \in W_{a, b}^{1} \cup H_{a, b}$

Combining the above lemma with Lemma 4.3, we obtain the following.

Theorem 4.14 Assume $m \in W_{a, b}^{1}$. Then there exists exactly one middle control point $z$ in $W_{\alpha,-\beta}^{0}$, and exactly one middle control point $z$ in $W_{\alpha,-\beta}^{1}$. 


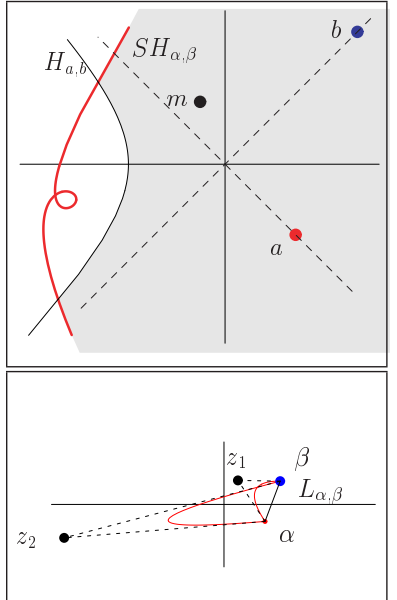

(a)

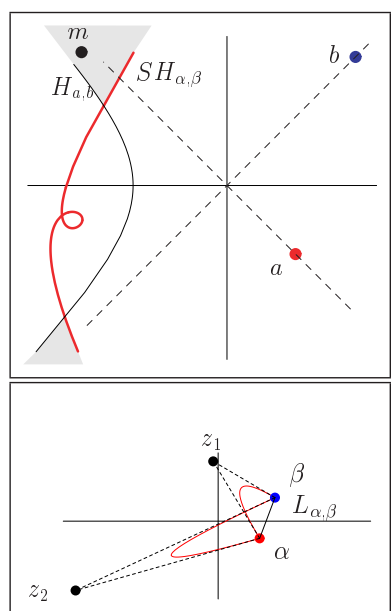

(b)

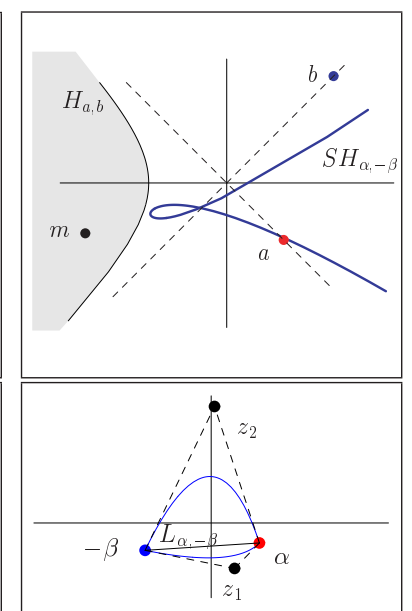

(c)

Figure 14: Illustration of Theorem 4.16 - (a): The case $m \in W_{a, b}^{0} \cap \overline{\mathcal{N}_{\alpha, \beta}^{1}}$ with $z_{1} \in W_{\alpha, \beta}^{0}$ and $z_{2} \in W_{\alpha, \beta}^{1} \cup H_{\alpha, \beta}$. (b): The case $m \in W_{a, b}^{0} \cap \mathcal{N}_{\alpha, \beta}^{2}$ with $z_{1} \in W_{\alpha, \beta}^{0}$ and $z_{2} \in W_{\alpha, \beta}^{0}$. (c): The case $m \in W_{a, b}^{1} \subset \mathcal{N}_{\alpha,-\beta}^{1}$ with $z_{1} \in W_{\alpha,-\beta}^{0}$ and $z_{2} \in W_{\alpha,-\beta}^{1}$.

Corollary 4.15 $S H_{\alpha,-\beta}$ does not meet $H_{a, b}$, and the island of $S H_{\alpha,-\beta}$ with its boundary lies entirely in the right side of $H_{a, b}$.

We are now ready to expound on the uniqueness/non-uniqueness question. First, assume $m \in W_{a, b}^{0}$, i.e., $m$ lies to the right of $H_{a, b}$. Then by Lemma 4.1 $s(t)$ goes from $\alpha$ to $\beta$. Therefore, we are confined to the three cases in Figure 12 . Combining with Lemma 4.12 reduces this to the two cases in Figure 14(a) and 14(b). Now the shaded region in Figure 14(a) lies to the right of $H_{a, b}$ and $S H_{\alpha, \beta}$. If $m$ lies in that region, one of the middle control points is in $W_{\alpha, \beta}^{0}$, and the other is in $W_{\alpha, \beta}^{1}$ from the first statement in Lemma 4.10. Hence, uniqueness holds there. (Note that when $m \in W_{a, b}^{0} \cap S H_{\alpha, \beta}$, it is clear that one middle control point is in $W_{\alpha, \beta}^{0}$, and the other in $H_{\alpha, \beta}$ by Lemma 4.3 and the definition of $S H_{\alpha, \beta}$. Thus, uniqueness also holds in $W_{a, b}^{0} \cap S H_{\alpha, \beta}$.) On the other hand, the shaded region depicted in Figure 14(b) lies to the right of $H_{a, b}$ but to the left of $S H_{\alpha, \beta}$ (or the upper/lower region of $S H_{\alpha, \beta}$ ). From the second and the third statements in Lemma 4.10, this region has the property that if $m$ lies there, both the middle control points $z$ are in $W_{\alpha, \beta}^{0}$. Therefore there are two solutions to the existence problem, which means that each represents the solution with zero hodograph winding number wind $\left(r^{\prime} \ominus \gamma^{\prime}\right)=0$. This is the region where the uniqueness theorem fails.

Assume now that $m \in W_{a, b}^{1}$, i.e., $m$ lies in the left side of $H_{a, b}$. Then by Lemma 4.1, $s(t)$ goes from $\alpha$ to $-\beta$. Therefore, we must examine the cases in 
Figure 13. However, Theorem 4.14 and Corollary 4.15 imply that there is only one possibility, namely, the case depicted in Figure 14(c). Therefore, in this case, the uniqueness theorem holds.

Theorem 4.16 Suppose $a, b, \alpha, \beta$ are in the form of the standard configuration. Then, $W_{a, b}^{0}$ is included in $\overline{\mathcal{N}_{\alpha, \beta}^{1}} \cup \mathcal{N}_{\alpha, \beta}^{2}$, and $W_{a, b}^{0} \cap \overline{\mathcal{N}_{\alpha, \beta}^{1}}$ and $W_{a, b}^{0} \cap \mathcal{N}_{\alpha, \beta}^{2}$ are non-empty sets. Also, $W_{a, b}^{1}$ is included in $\mathcal{N}_{\alpha,-\beta}^{1}$.

(i) If $m \in W_{a, b}^{0} \cap \overline{\mathcal{N}_{\alpha, \beta}^{1}}$, there exists a unique quintic PH Hermite interpolant $\gamma(t)$ such that wind $\left(r^{\prime} \ominus \gamma^{\prime}\right)=0$.

(ii) If $m \in W_{a, b}^{0} \cap \mathcal{N}_{\alpha, \beta}^{2}$, there exist two quintic PH Hermite interpolants $\gamma(t)$ such that wind $\left(r^{\prime} \ominus \gamma^{\prime}\right)=0$.

(iii) If $m \in W_{a, b}^{1}$ there exists a unique PH Hermite interpolant $\gamma(t)$ such that $\operatorname{wind}\left(r^{\prime} \ominus \gamma^{\prime}\right)=0$.

\subsection{Analytic continuation}

Consider first the case in which uniqueness fails, i.e., the case where $m \in W_{a, b}^{0} \cap$ $\mathcal{N}_{\alpha, \beta}^{2}$ (depicted in Figure 14(b)). In this case, both the middle control points $z$ of $\chi(t)$ are in $W_{\alpha, \beta}^{0}$, thereby making wind $(\chi \ominus s)=0$ for either case. On the other hand, when $m \in W_{a, b}^{0} \cap \overline{\mathcal{N}_{\alpha, \beta}^{1}}$, one middle control point $z$ of $\chi(t)$ is in $W_{\alpha, \beta}^{0}$ and the other in $W_{\alpha, \beta}^{1} \cup H_{\alpha, \beta}$ (see Figure 14(a)). Hence, in this case, uniqueness holds.

The relation between these cases can be explained as follows. As $m$ moves from $W_{a, b}^{0} \cap \overline{\mathcal{N}_{\alpha, \beta}^{1}}$ to $W_{a, b}^{0} \cap \mathcal{N}_{\alpha, \beta}^{2}$, each of two branches of $z$ is analytically continued. Thus, it is advantageous to choose the value (branch) of $z$ obtained by the analytic continuation of $z$ that gives uniqueness. This idea can be precisely formulated in the following theorem, whose proof can be found in the Appendix.

Theorem 4.17 Suppose $m \in W_{a, b}^{0} \cup H_{a, b}$. Then, any square root of

$$
\frac{5}{4}\left(6 d-a-b+\frac{1}{4}(\alpha+\beta)^{2}\right)
$$

has a non-zero real part. Let $K$ be the square root with positive real part. Then $z=K-\frac{3}{4}(\alpha+\beta)$ is the middle control point of $\chi(t)$ from $\alpha$ to $\beta$ such that $z \in W_{\alpha, \beta}^{0}$. Thus if $m \in W_{a, b}^{0}$, wind $\left(r^{\prime} \ominus \gamma^{\prime}\right)=0$, where $\gamma^{\prime}(t)=\left[\alpha(1-t)^{2}+\right.$ $\left.z 2(1-t) t+\beta t^{2}\right]^{2}$.

On the other hand, we know that when $m \in W_{a, b}^{1}$ uniqueness holds as shown in Figure 14(c). However, it is still important in practice to have a formula for $z$. The following theorem, whose proof can be found in Appendix, gives such a $z$.

Theorem 4.18 Suppose $m \in W_{a, b}^{1} \cup H_{a, b}$. Then, any square root of

$$
\frac{5}{4}\left(6 d-a-b+\frac{1}{4}(\alpha-\beta)^{2}\right)
$$




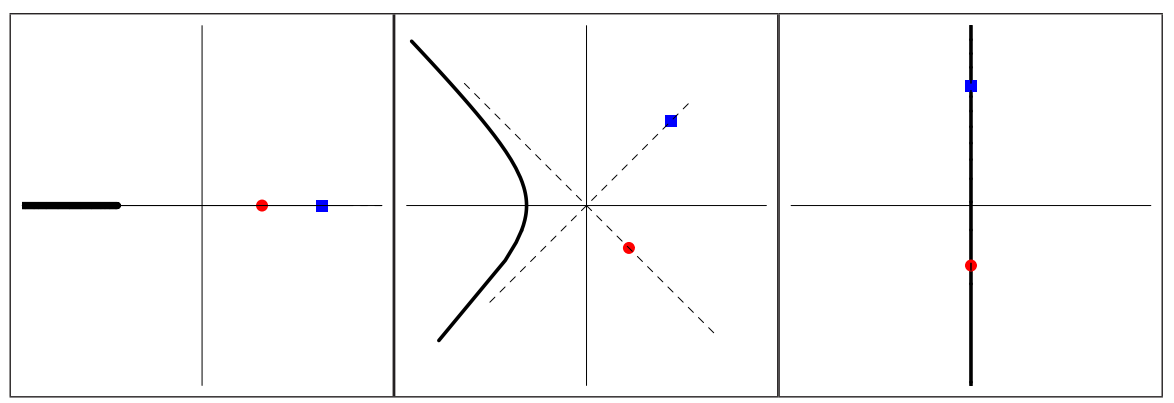

Figure 15: $H_{a, b}$ where $a=r_{1} e^{-i \theta}, b=r_{2} e^{i \theta}$ and $\theta=0 ; 0<\theta<\frac{1}{2} \pi ; \theta=\frac{1}{2} \pi$.

has non-zero imaginary part. Let $K$ be the root with $\sin \frac{1}{2} \theta \operatorname{Im} K<0$. Then $z=K-\frac{3}{4}(\alpha-\beta)$ is the middle control point of $\chi(t)$ from $\alpha$ to $-\beta$ such that $z \in W_{\alpha,-\beta}^{0}$. Thus if $m \in W_{a, b}^{1}$, wind $\left(r^{\prime} \ominus \gamma^{\prime}\right)=0$, where $\gamma^{\prime}(t)=\left[\alpha(1-t)^{2}+\right.$ $\left.z 2(1-t) t-\beta t^{2}\right]^{2}$.

Analytic Continuation on $H_{a, b}$ : From the above two theorems, each of the two solutions - one obtained when $m \in W_{a, b}^{0}$ and the other when $m \in W_{a, b}^{1}$ can be analytically continued to the solutions when $m \in H_{a, b}$. When $m \in H_{a, b}$, $r^{\prime}(t)$ passes through the origin; thus wind $\left(r^{\prime} \ominus \gamma^{\prime}\right)$ is undefined. However, these two continued solutions on $H_{a, b}$ do not pass through the origin.

\section{Non-generic case}

The case not considered above is the one in which $a, b$ are collinear. Although the direct argument for this case can be complicated, the easier approach is to treat it as a limiting case of the cases considered above. Here we present an intuitive limiting argument for this case (a rigorous proof is in the Appendix).

\subsection{Case 1: $a$ and $b$ are in the same direction}

By a rotation, we may assume $a$ and $b$ are positive real numbers, $r_{1}$ and $r_{2}$. This case can be thought as the limiting case of $a_{\theta} \rightarrow a=r_{1}$ and $b_{\theta} \rightarrow b=r_{2}$, where $a_{\theta}=r_{1} e^{-i \theta}$ and $b_{\theta}=r_{2} e^{i \theta}$. Then it is easy to see that, as $\theta \rightarrow 0$,

$$
H_{a_{\theta}, b_{\theta}} \rightarrow H_{r_{1}, r_{2}}=\left(-\infty,-\sqrt{r_{1} r_{2}} .\right.
$$

Let $\alpha_{\theta}=\sqrt{r_{1}} e^{-i \theta / 2}, \beta_{\theta}=\sqrt{r_{2}} e^{i \theta / 2}$ and $\alpha=\sqrt{r_{1}}, \beta=\sqrt{r_{2}}$. Then it is also easy to see that as $\theta \rightarrow 0$

$$
S H_{\alpha_{\theta}, \beta_{\theta}} \rightarrow S H_{\sqrt{r_{1}}, \sqrt{r_{2}}}=[-L, \infty),
$$

where $L$ is $\frac{1}{8}\left(5 r_{1}+5 r_{2}+2 \sqrt{r_{1} r_{2}}\right)$ (note that $\left.-L<-\sqrt{r_{1} r_{2}}\right)$.

Thus the shaded region in Figure $14(\mathrm{~b})$ converges to the open set $\mathbb{C}-$ $\{x$-axis $\}$, the shaded region in Figure $14(\mathrm{a})$ disappears, and the hyperbola 
$H_{a, b}$ collapses into the line $\left(-\infty,-\sqrt{r_{1} r_{2}}\right]$ while $S H_{\alpha, \beta}$ collapses into the line $[-L, \infty)$. Also, $\psi_{\alpha, \beta}:\left(-\infty,-\sqrt[4]{r_{1} r_{2}}\right] \rightarrow[-L, \infty)$ is the quadratic function

$$
\psi_{\alpha, \beta}(z)=\frac{2}{5}\left(z+\frac{3}{4}\left(\sqrt{r_{1}}+\sqrt{r_{2}}\right)\right)^{2}-\frac{1}{2} r_{1}-\frac{1}{2} r_{2}-\frac{1}{8}\left(\sqrt{r_{1}}+\sqrt{r_{2}}\right)^{2} .
$$

It is easy to see that $\phi_{\alpha, \beta}$ has two inverses (with multiplicity) if and only if $m \in[-L,-S]$, where $-S=\phi_{\alpha, \beta}\left(-\sqrt[4]{r_{1} r_{2}}\right)$ and $-L<-S<-\sqrt{r_{1} r_{2}}$. Therefore we have the following theorem.

\section{Theorem 5.1}

(i) $r^{\prime}(t)$ passes through the origin if and only if $m$ lies in the semi-infinite line $\left(-\infty,-\sqrt{r_{1} r_{2}}\right]$. In this case, $\operatorname{wind}\left(r^{\prime} \ominus \gamma^{\prime}\right)$ is undefined.

(ii) If $m$ lies in $\mathbb{C}-\{x$-axis $\}$, then there are two solutions $\gamma$ such that the hodograph winding number $\operatorname{wind}\left(r^{\prime} \ominus \gamma^{\prime}\right)=0$.

(iii) If $m$ lies on the half infinite line $\left(-\sqrt{r_{1} r_{2}}, \infty\right)$ on the $x$-axis, then there is the unique solution $\gamma$ such that $\operatorname{wind}\left(r^{\prime} \ominus \gamma^{\prime}\right)=0$. In this case, the hodograph of the other solution passes through the origin.

The "good" solution selection formula can be obtained by taking the limiting value - we can easily see that it is the same as in Theorem 4.17.

Theorem 5.2 Suppose $a=r_{1}, b=r_{2}$ for positive real values $r_{1}, r_{2}$ and write $\alpha=\sqrt{r_{1}}, \beta=\sqrt{r_{2}}$. If $m \notin H_{a, b}=\left(-\infty,-\sqrt{r_{1} r_{2}}\right]$, any square root of

$$
\frac{5}{4}\left(6 d-a-b+\frac{1}{4}(\alpha+\beta)^{2}\right)
$$

has a non-zero real part. Let $K$ be the square root with $\operatorname{Re}(K)>0$. Then

$$
z=K-\frac{3}{4}(\alpha+\beta)
$$

is the middle control point of $\chi(t)$ from $\alpha$ to $\beta$ such that $\operatorname{wind}\left(r^{\prime} \ominus \gamma^{\prime}\right)=0$, where $\gamma^{\prime}(t)=\left[\alpha(1-t)^{2}+z 2(1-t) t+\beta t^{2}\right]^{2}$.

\subsection{Case 2: $a$ and $b$ are in opposite directions}

We may assume $a=r_{1} e^{-i \pi / 2}, b=r_{2} e^{i \pi / 2}$ for some positive $r_{1}, r_{2}$ and we set $\alpha=\sqrt{r_{1}} e^{-i \pi / 4}, \beta=\sqrt{r_{2}} e^{i \pi / 4}$ and define $a_{\theta}=r_{1} e^{-i \theta}, b_{\theta}=r_{2} e^{i \theta}$. To take the limit, we let $\theta \rightarrow \frac{1}{2} \pi$, so that $a_{\theta} \rightarrow a, b_{\theta} \rightarrow b$. It is then easy to verify that

$$
H_{a_{\theta}, b_{\theta}} \rightarrow H_{a, b}=\{y \text {-axis }\} \quad \text { and } \quad S H_{\alpha_{\theta}, \pm \beta_{\theta}} \rightarrow S H_{\alpha, \pm \beta},
$$

where $\operatorname{Re}\left(S H_{\alpha, \beta}\right)<0$ and $\operatorname{Re}\left(S H_{\alpha,-\beta}\right)>0\left(S H_{\alpha, \beta}\right.$ and $S H_{\alpha,-\beta}$ are symmetric with respect to the $y$-axis). In fact, the shaded region in Figure 14(a) converges to the right half-plane $\operatorname{Re} z>0$, the shaded region in Figure 14(b) disappears, and the shaded region in Figure 14(c) converges to the left half-plane $\operatorname{Re} z<0$. Thus, we have the following theorem. 
Theorem 5.3 Let $a, b$ be as defined above. Then

(i) $r^{\prime}(t)$ passes through the origin if and only if $m$ lies on the $y$-axis. In this case, wind $\left(r^{\prime} \ominus \gamma^{\prime}\right)$ is undefined.

(ii) If $m$ lies in the open set $\mathbb{C}-\{y$-axis $\}$, there exists a unique solution $\gamma$ such that $\operatorname{wind}\left(r^{\prime} \ominus \gamma^{\prime}\right)=0$.

However, the selection formula can also be obtained by a limiting argument applied to the formula given in Section 4, and this differs depending on whether $m$ lies in the right half plane or in the left half plane. In fact, the selection formula when $m$ is in the right half-plane comes from Theorem 4.17 and when $m$ is in the left half-plane it comes from Theorem 4.18 with $\theta=\frac{1}{2} \pi$. The selection formula for the "good" solution is thus given in the following proposition.

Proposition 5.4 Let $a, b, \alpha, \beta$ be as defined above.

(i) If $\operatorname{Re}(m) \geq 0$, any square root of

$$
\frac{5}{4}\left(6 d-a-b+\frac{1}{4}(\alpha+\beta)^{2}\right)
$$

has non-zero real part. Let $K$ be the root with positive real part. Then $z=K-\frac{3}{4}(\alpha+\beta)$ is the middle control point of $\chi(t)$ from $\alpha$ to $\beta$ such that $z \in W_{\alpha, \beta}^{0}$. Therefore, if $\operatorname{Re}(m)>0$, wind $\left(r^{\prime} \ominus \gamma^{\prime}\right)=0$ where $\gamma^{\prime}(t)=$ $\left[\alpha(1-t)^{2}+z 2(1-t) t+\beta t^{2}\right]^{2}$.

(ii) If $\operatorname{Re}(m) \leq 0$, any square root of

$$
\frac{5}{4}\left(6 d-a-b+\frac{1}{4}(\alpha-\beta)^{2}\right)
$$

has non-zero imaginary part. Let $K$ be the root with negative imaginary part. Then $z=K-\frac{3}{4}(\alpha-\beta)$ is the middle control point of $\chi(t)$ from $\alpha$ to $-\beta$ such that $z \in W_{\alpha,-\beta}^{0}$. Therefore, if $\operatorname{Re}(m)<0$, $\operatorname{wind}\left(r^{\prime} \ominus \gamma^{\prime}\right)=0$ where $\gamma^{\prime}(t)=\left[\alpha(1-t)^{2}+z 2(1-t) t-\beta t^{2}\right]^{2}$.

Analytic Continuation on $y$-axis: Similar analytic continuation can be applied as in Section 4.3. From the above proposition, each of the two solutions - one obtained when $\operatorname{Re}(m)>0$ and the other obtained when $\operatorname{Re}(m)<0$ - can be analytically continued to the solution when $\operatorname{Re}(m)=0$. When $\operatorname{Re}(m)=0$, $r^{\prime}(t)$ passes through the origin; thus wind $\left(r^{\prime} \ominus \gamma^{\prime}\right)$ is not defined. However, these two continued solutions on the $y$-axis do not pass through the origin.

\section{Best selection formula}

For numbers $r_{1}>0, r_{2}>0,-\frac{\pi}{2}<\theta \leq \frac{\pi}{2}$, let $a=r_{1} e^{-i \theta}, b=r_{2} e^{i \theta}, \alpha=$ $\sqrt{r_{1}} e^{-i \theta / 2}, \beta=\sqrt{r_{2}} e^{i \theta / 2}$. For given complex number $d \in \mathbb{C}$, let $r(t)$ be the cubic Bézier curve such that

$$
r^{\prime}(t)=a(1-t)^{2}+m 2(1-t) t+b t^{2}
$$


where $m=3 d-a-b$. Suppose $r^{\prime}(t) \neq 0$ for all $t \in[0,1]$. This condition is equivalent to that $m \notin H_{a, b}$. Let $\gamma(t)$ be the quintic $\mathrm{PH}$ curve such that

$$
\gamma^{\prime}(t)=\chi(t)^{2},
$$

$\chi(t)$ being given by the selection rule below. Then, $\gamma^{\prime}(t) \neq 0$ for $t \in[0,1]$ and

$$
\operatorname{wind}\left(r^{\prime} \ominus \gamma^{\prime}\right)=0 \text {. }
$$

\section{Selection rule: standard configuration}

(i) $(\theta=0)$ or $\left(0<|\theta|<\pi / 2\right.$ and $\left.m \in W_{a, b}^{0}\right)$ or $(\theta=\pi / 2$ and $\operatorname{Re}(m)>0)$

Let $K$ be the square root of

$$
\frac{5}{4}\left(6 d-a-b+\frac{1}{4}(\alpha+\beta)^{2}\right)
$$

such that $\operatorname{Re}(K)>0$, and let $z=K-\frac{3}{4}(\alpha+\beta)$. Then we select $\chi(t)=$ $\alpha(1-t)^{2}+z 2(1-t) t+\beta t^{2}$.

(ii) $\left(0<|\theta|<\pi / 2\right.$ and $\left.m \in W_{a, b}^{1}\right)$ or $(\theta=\pi / 2$ and $\operatorname{Re}(m)<0)$

Let $K$ be the square root of

$$
\frac{5}{4}\left(6 d-a-b+\frac{1}{4}(\alpha-\beta)^{2}\right)
$$

such that $\sin \frac{1}{2} \theta \operatorname{Im}(K)<0$, and let $z=K-\frac{3}{4}(\alpha-\beta)$. Then we select $\chi(t)=\alpha(1-t)^{2}+z 2(1-t) t-\beta t^{2}$.

Suppose we are given non-zero $a, b$ not necessarily in standard configuration. Also, suppose that we are given $d$ such that $m=3 d-a-b \notin H_{a, b}$. For a non-zero complex number $z$, let $\operatorname{Arg} z$ be the argument of $z$ such that $-\pi<\operatorname{Arg} z \leq \pi$. First, we need to find $\theta_{0}$ and $\theta$ :

(i) Find $\theta_{0}$ such that $a=|a| e^{i \theta_{0}}$ (Not necessarily $\theta_{0}=\operatorname{Arg} a$ )

(ii) Find $\theta$ such that $2 \theta=\operatorname{Arg}(b / a)$

Now, writing $\alpha=\sqrt{|a|} e^{i \theta_{0} / 2}, \beta=\sqrt{|b|} e^{i\left(\theta_{0} / 2+\theta\right)}$, and $R=e^{-i\left(\theta_{0}+\theta\right) / 2}$, we need only follow the scheme below, which is easy to verify.

\section{Selection rule}

(i) $(\theta=0)$ or $\left(0<|\theta|<\pi / 2\right.$ and $\left.m \in W_{a, b}^{0}\right)$ or $\left(\theta=\pi / 2\right.$ and $\left.\operatorname{Re}\left(m R^{2}\right)>0\right)$ Let $K$ be the square root of

$$
\frac{5}{4}\left(6 d-a-b+\frac{1}{4}(\alpha+\beta)^{2}\right)
$$

such that $\operatorname{Re}(K R)>0$. Let $z$ be the complex number obtained by

$$
z=K-\frac{3}{4}(\alpha+\beta) .
$$

Then we select $\chi(t)=\alpha(1-t)^{2}+z 2(1-t) t+\beta t^{2}$. 
(ii) $\left(0<|\theta|<\pi / 2\right.$ and $\left.m \in W_{a, b}^{1}\right)$ or $\left(\theta=\pi / 2\right.$ and $\left.\operatorname{Re}\left(m R^{2}\right)<0\right)$

Let $K$ be the square root of

$$
\frac{5}{4}\left(6 d-a-b+\frac{1}{4}(\alpha-\beta)^{2}\right)
$$

such that $\sin \frac{1}{2} \theta \operatorname{Im}(K R)<0$. Let $z$ be the complex number obtained by

$$
z=K-\frac{3}{4}(\alpha-\beta) .
$$

Then we select $\chi(t)=\alpha(1-t)^{2}+z 2(1-t) t-\beta t^{2}$.

\section{Closure}

A topological criterion for identifying the "good" solution among the four PH quintic interpolants to planar Hermite data has been proposed. The analysis includes an existence theorem, together with a complete discussion of uniqueness properties. The availability of a simple formula for finding the "good" solution, without appealing to curve fairness integrals, is a key step in making practical use of the many advantageous computational properties of $\mathrm{PH}$ curves.

\section{Appendix 1: Non-generic cases}

\subsection{Case 1: $a$ and $b$ are in the same direction}

Suppose that $a, b \neq 0$ and $b=\lambda a$ for some $\lambda>0$. Without loss of generality, we can assume that $a=r_{1}, b=r_{2}$ for some $r_{1}, r_{2}>0$. In this case, $H_{r_{1}, r_{2}}=$ $\left(-\infty,-\sqrt{r_{1} r_{2}}\right]$ from Lemma 8.1 .

Lemma 8.1 For positive real numbers $r_{1}, r_{2}$ set $H_{r_{1}, r_{2}}=\left(-\infty,-\sqrt{r_{1} r_{2}}\right]$. For a complex number $\mu \in \mathbb{C}$, let $Q(t)=r_{1}(1-t)^{2}+\mu 2(1-t) t+r_{2} t^{2}$. Then if $\mu \notin H_{r_{1}, r_{2}}$, the angle variation $\Delta \theta_{Q}$ is zero.

Proof. From the definition of $H_{r_{1}, r_{2}}$,

$$
H_{r_{1}, r_{2}}=\left\{\lambda_{1} r_{1}+\lambda_{2} r_{2} \mid \lambda_{1}<0, \lambda_{2}<0, \lambda_{1} \lambda_{2}=\frac{1}{4}\right\},
$$

we need to get find range of the function $f(t)=r_{1} t+r_{2} / 4 t$, where $t<0$. Clearly, for all $t<0, f(t)<0$ and

$$
r_{1} t+r_{2} / 4 t \leq-\sqrt{r_{1} r_{2}} .
$$

Moreover, since $\lim _{t \rightarrow-\infty} f(t)=-\infty$,

$$
H_{r_{1}, r_{2}}=\left(-\infty,-\sqrt{r_{1} r_{2}}\right] .
$$

If $\mu \notin H_{r_{1}, r_{2}}$, then $Q(t) \neq 0$ for all $t \in[0,1]$ by Theorem 3.2. Then, $Q(t) \notin$ $(-\infty, 0]$ for all $t \in[0,1]$ from the argument below. 
(i) $\operatorname{Im}(\mu)=0$ : In this case, $Q(t)$ is real for $t \in[0,1]$. If $Q\left(t_{0}\right)<0$ for some $t_{0} \in[0,1]$, then by the Intermediate Value Theorem there exists a $t_{1} \in(0,1)$ such that $Q\left(t_{1}\right)=0$. Therefore, $Q(t) \notin(-\infty, 0]$ for all $t \in[0,1]$.

(ii) $\operatorname{Im}(\mu) \neq 0$ : Since $\operatorname{Im} Q(t)=(1-t) t \operatorname{Im}(\mu), \operatorname{Im} Q(t) \neq 0$ for all $t \in(0,1)$. Thus, $Q(t) \notin(-\infty, 0]$.

Therefore, $\Delta \theta_{Q}=\operatorname{Arg}\left(r_{2}\right)-\operatorname{Arg}\left(r_{1}\right)=0$.

Now suppose that $m=3 d-a-b \notin H_{r_{1}, r_{2}}$. Let $\alpha=\sqrt{r_{1}}, \beta=\sqrt{r_{2}}$. Let $s(t)$ be the square-root curve of $r^{\prime}(t)$ such that $s(0)=\alpha$. By Lemma 8.1, $\Delta \theta_{r^{\prime}}=0$. Therefore, $s(1)=\beta$. So, by Lemma 3.5, we need to consider only $\chi(t)=\alpha(1-t)^{2}+z 2(1-t) t+\beta t^{2}$ with $z$ satisfying

$$
\left(z+\frac{3}{4}(\alpha+\beta)\right)^{2}=\frac{5}{4}\left(6 d-r_{1}-r_{2}+\frac{1}{4}(\alpha+\beta)^{2}\right) .
$$

Let $L_{\alpha, \beta}=\alpha(1-t)+\beta t$ be the line segment connecting $\alpha$ and $\beta$. Note that if $\chi(t) \neq 0$ for all $t \in[0,1]$,

$$
\operatorname{wind}\left(r^{\prime} \ominus \chi^{2}\right)=2 \operatorname{wind}(s \ominus \chi)=2 \operatorname{wind}\left(L_{\alpha, \beta} \ominus \chi\right)
$$

since $\Delta \theta_{s}=\Delta \theta_{L_{\alpha, \beta}}=0$. Thus, we need to investigate when there exists at least one solution $z$ of (17) such that $\operatorname{wind}\left(L_{\alpha, \beta} \ominus \chi\right)=0$. By Lemma 8.1, this is equivalent to that $z \notin H_{\alpha, \beta}=\left(-\infty,-\sqrt[4]{r_{1} r_{2}}\right]$.

Let us choose the square root $K$ of the right hand side of (17) such that $\operatorname{Re}(K)>0$. This is possible from the following Lemma.

Lemma 8.2 Let $r_{1}, r_{2}$ be positive real numbers, and let $\alpha=\sqrt{r_{1}}, \beta=\sqrt{r_{2}}$. Suppose $m=3 d-r_{1}-r_{2} \notin H_{r_{1}, r_{2}}$. Let $K$ be a square root of

$$
\frac{5}{4}\left(6 d-r_{1}-r_{2}+\frac{1}{4}(\alpha+\beta)^{2}\right) .
$$

Then, $\operatorname{Re}(K) \neq 0$.

Proof. Suppose $\operatorname{Re}(K)=0$. Then $K^{2} \leq 0$, i.e.,

$$
6 d-r_{1}-r_{2}+\frac{1}{4}(\alpha+\beta)^{2} \leq 0 .
$$

Thus, we get the following inequality

$$
6 d-2 r_{1}-2 r_{2} \leq-r_{1}-r_{2}-\frac{1}{4}(\alpha+\beta)^{2} \leq-2 \sqrt{r_{1} r_{2}}-\alpha \beta=-3 \sqrt{r_{1} r_{2}} .
$$

This is a contradiction to $m=3 d-r_{1}-r_{2} \notin H_{r_{1}, r_{2}}=\left(-\infty,-\sqrt{r_{1} r_{2}}\right]$.

Then, two solutions $z_{1}$ and $z_{2}$ of Equation (17) can be written as

$$
z_{1}=K-\frac{3}{4}\left(\sqrt{r_{1}}+\sqrt{r_{2}}\right), \quad z_{2}=-K-\frac{3}{4}\left(\sqrt{r_{1}}+\sqrt{r_{2}}\right) .
$$

Theorem 8.3 Let $\chi(t)=\alpha(1-t)^{2}+z_{1} 2(1-t) t+\beta t^{2}$, with $z_{1}$ given by (18). Let $\gamma(t)$ be a PH quintic with $\gamma^{\prime}(t)=\chi(t)^{2}$. If $m \notin H_{r_{1}, r_{2}}=\left(-\infty,-\sqrt{r_{1} r_{2}}\right], r^{\prime}(t) \neq$ 0 and $\gamma^{\prime}(t) \neq 0$ for all $t \in[0,1]$ and $\operatorname{wind}\left(r^{\prime} \ominus \gamma^{\prime}\right)=0$. If $m \in\left(-\sqrt{r_{1} r_{2}}, \infty\right), z_{1}$ is the unique solution such that $\operatorname{wind}\left(r^{\prime} \ominus \gamma^{\prime}\right)=0$. If $\operatorname{Im}(m) \neq 0$, both $z_{1}$ and $z_{2}$ are such solutions that wind $\left(r^{\prime} \ominus \gamma^{\prime}\right)=0$. 


\section{Proof.}

(i) $m \in\left(-\sqrt{r_{1} r_{2}},+\infty\right)$

In this case, since the right hand side of (17) is a real number and $3 d>$ $r_{1}+r_{2}-\sqrt{r_{1} r_{2}}$,

$$
\begin{aligned}
K^{2} & =\frac{5}{4}\left(6 d-r_{1}-r_{2}+\frac{1}{4}(\alpha+\beta)^{2}\right) \\
& >\frac{5}{4}\left(r_{1}+r_{2}-2 \sqrt{r_{1} r_{2}}+\frac{1}{4}(\alpha+\beta)^{2}\right) \\
& =\frac{5}{4}\left(\left(\sqrt{r_{1}}-\sqrt{r_{2}}\right)^{2}+\frac{1}{4}\left(\sqrt{r_{1}}+\sqrt{r_{2}}\right)^{2}\right) .
\end{aligned}
$$

So, $K$ is a positive real number. Then, since

$$
-\frac{3}{4}\left(\sqrt{r_{1}}+\sqrt{r_{2}}\right) \leq-\frac{3}{2} \sqrt[4]{r_{1} r_{2}},
$$

$z_{2}<-\sqrt[4]{r_{1} r_{2}}$, i.e., $z_{2} \in H_{\alpha, \beta}=\left(-\infty,-\sqrt[4]{r_{1} r_{2}}\right)$. So, $\chi(t)=0$ for some $t \in(0,1)$, where $\chi(t)$ is constructed by using $z_{2}$. Now let us show that $z_{1}>-\sqrt[4]{r_{1} r_{2}}$. It suffices to verify that

$$
K^{2}>\left(\frac{3}{4}\left(\sqrt{r_{1}}+\sqrt{r_{2}}\right)-\sqrt[4]{r_{1} r_{2}}\right)^{2} .
$$

This fact comes from the following inequalities.

$$
\begin{aligned}
& K^{2}-\left(\frac{3}{4}\left(\sqrt{r_{1}}+\sqrt{r_{2}}\right)-\sqrt[4]{r_{1} r_{2}}\right)^{2} \\
& \quad>\frac{5}{4}\left(\left(\sqrt{r_{1}}-\sqrt{r_{2}}\right)^{2}+\frac{1}{4}\left(\sqrt{r_{1}}+\sqrt{r_{2}}\right)^{2}\right)-\left(\frac{3}{4}\left(\sqrt{r_{1}}+\sqrt{r_{2}}\right)-\sqrt[4]{r_{1} r_{2}}\right)^{2} \\
& \quad=-\frac{1}{4}\left(\sqrt{r_{1}}+\sqrt{r_{2}}\right)^{2}+\frac{5}{4}\left(\sqrt{r_{1}}-\sqrt{r_{2}}\right)^{2}+\frac{3}{2}\left(\sqrt{r_{1}}+\sqrt{r_{2}}\right) \sqrt[4]{r_{1} r_{2}}-\sqrt{r_{1} r_{2}} \\
& \quad=r_{1}+r_{2}-4 \sqrt{r_{1} r_{2}}+\frac{3}{2}\left(\sqrt{r_{1}}+\sqrt{r_{2}}\right) \sqrt[4]{r_{1} r_{2}} \\
& \quad=\left(\sqrt{r_{1}}-\sqrt{r_{2}}\right)^{2}-2 \sqrt{r_{1} r_{2}}+\frac{3}{2}\left(\sqrt{r_{1}}+\sqrt{r_{2}}\right) \sqrt[4]{r_{1} r_{2}} \\
& \quad>\left(\sqrt{r_{1}}-\sqrt{r_{2}}\right)^{2}-2 \sqrt{r_{1} r_{2}}+3 \sqrt{r_{1} r_{2}}=\left(\sqrt{r_{1}}-\sqrt{r_{2}}\right)^{2}+\sqrt{r_{1} r_{2}} .
\end{aligned}
$$

So, $z_{1} \notin H_{\alpha, \beta}$. Therefore, the angle variation is $\Delta \theta_{\chi}=0$, where $\chi(t)$ is constructed by using $z_{1}$. Thus, $\operatorname{wind}\left(L_{\alpha, \beta} \ominus \chi\right)=0$.

(ii) $\operatorname{Im}(m) \neq 0$

In this case, $\operatorname{Im}\left(K^{2}\right) \neq 0$ since $\operatorname{Im}(d) \neq 0$. So, $z_{1}, z_{2}$ are non-real numbers. Thus, $z_{1} \notin H_{\alpha, \beta}$ and $z_{2} \notin H_{\alpha, \beta}$. So, by Lemma 8.1 , the angle variation $\Delta \theta_{\chi}$ is well defined, and $\Delta \theta_{\chi}=0$, where $\chi(t)$ is constructed by using $z_{1}$ or $z_{2}$. Thus, wind $\left(L_{\alpha, \beta} \ominus \chi\right)=0$.

In any case, $z_{1} \notin H_{\alpha, \beta}$ by the above argument. The angle variation $\Delta \theta_{\chi}$ of $\chi(t)=\alpha(1-t)^{2}+z_{1} 2(1-t) t+\beta t^{2}$ is thus well defined, and clearly $\Delta \theta_{\chi}=0$ from Lemma 8.1. Thus, the winding number, $\operatorname{wind}\left(L_{\alpha, \beta} \ominus \chi\right)=0$.

Although $z_{2}$ also gives zero winding number solution in some cases, the curve shape obtained from $z_{1}$ is better than that from $z_{2}$. 


\subsection{Case 2: $a$ and $b$ are in the opposite direction}

Suppose that $a, b \neq 0$ and $b=\lambda a$ for some $\lambda<0$. Without loss of generality, we can set $a=r_{1} e^{-i \pi / 2}, b=r_{2} e^{i \pi / 2}$ for some $r_{1}, r_{2}>0$. Let $\alpha=\sqrt{r_{1}} e^{-i \pi / 4}$, $\beta=\sqrt{r_{2}} e^{i \pi / 4}$. In this case $H_{a, b}=\{y i \mid y \in \mathbb{R}\}$ from Lemma 8.4.

Lemma 8.4 Let $a=-r_{1} i$ and $b=r_{2} i$ for positive real numbers $r_{1}, r_{2}$. Then $H_{a, b}=\{y i \mid y \in \mathbb{R}\}=\mathbb{R} i$. Let $Q(t)=a(1-t)^{2}+\mu 2(1-t) t+b t^{2}$ for some $\mu \in \mathbb{C}$. If $\operatorname{Re}(\mu)>0$ then $\Delta \theta_{Q}=\pi$, and if $\operatorname{Re}(\mu)<0$ then $\Delta \theta_{Q}=-\pi$.

Proof. From the definition $H_{a, b}=\left\{\left(-\lambda_{1} r_{1}+\lambda_{2} r_{2}\right) i \mid \lambda_{1}<0, \lambda_{2}<0, \lambda_{1} \lambda_{2}=\frac{1}{4}\right\}$ we need to determine range of the function $f(t)=-r_{1} t+r_{2} / 4 t$, where $t<0$. Since $\lim _{t \rightarrow-\infty} f(t)=\infty$ and $\lim _{t \rightarrow 0^{-}} f(t)=-\infty$, the range of $f(t)$ is $\mathbb{R}$. Thus, $H_{a, b}=\{y i \mid y \in \mathbb{R}\}=\mathbb{R} i$. Note that $Q(0)=-r_{1} i$ and $Q(1)=r_{2} i$. If $\operatorname{Re}(\mu)>0$, $\operatorname{Re} Q(t)>0$ for all $t \in(0,1)$ and hence $\Delta \theta_{Q}=\pi$. If $\operatorname{Re}(\mu)<0, \operatorname{Re} Q(t)<0$ for all $t \in(0,1)$ and hence $\Delta \theta_{Q}=-\pi$.

So $r^{\prime}(t)=0$ for some $t \in(0,1)$ if and only if $m=3 d-a-b \in \mathbb{R} i$. So, we assume that $\operatorname{Re}(m) \neq 0$. Since $a, b$ are pure imaginary values, this assumption is equivalent to $\operatorname{Re}(d) \neq 0$.

Theorem 8.5 If $\operatorname{Re}(m) \neq 0$ there is a unique solution $\chi(t)$ with $\operatorname{wind}\left(r^{\prime} \ominus \chi^{2}\right)=$ 0, obtained as follows:

(i) $\operatorname{Re}(m)>0$ : Let $K$ be the square root of $\frac{5}{4}\left(6 d-a-b+\frac{1}{4}(\alpha+\beta)^{2}\right)$ with $\operatorname{Re}(K)>0$, and $z=K-\frac{3}{4}(\alpha+\beta)$. Then $\chi(t)=\alpha(1-t)^{2}+z 2(1-t) t+\beta t^{2}$.

(ii) $\operatorname{Re}(m)<0$ : Let $K$ be the square root of $\frac{5}{4}\left(6 d-a-b+\frac{1}{4}(\alpha-\beta)^{2}\right)$ with $\operatorname{Im}(K)<0$, and $z=K-\frac{3}{4}(\alpha-\beta)$. Then $\chi(t)=\alpha(1-t)^{2}+z 2(1-t) t-\beta t^{2}$.

\section{Proof.}

(i) $\operatorname{Re}(m)>0$ : From Lemma 8.4, $\Delta \theta_{r^{\prime}}=\pi$ and if $s(0)=\alpha$, then $s(1)=\beta$. By Lemma 3.5, we need consider only $\chi(t)=\alpha(1-t)^{2}+z 2(1-t) t+\beta t^{2}$ with $z$ satisfying

$$
\left(z+\frac{3}{4}(\alpha+\beta)\right)^{2}=\frac{5}{4}\left(6 d-a-b+\frac{1}{4}(\alpha+\beta)^{2}\right) .
$$

Consider the line segment $L_{\alpha, \beta}=\alpha(1-t)+\beta t$. Since $\Delta \theta_{s}=\Delta \theta_{L_{\alpha, \beta}}=\frac{1}{2} \pi$,

$$
\operatorname{wind}\left(r^{\prime} \ominus \chi^{2}\right)=2 \operatorname{wind}(s \ominus \chi)=2 \operatorname{wind}\left(L_{\alpha, \beta} \ominus \chi\right) .
$$

We need to show that a solution $z$ of (19) exists so that wind $\left(L_{\alpha, \beta} \ominus \chi\right)=0$.

Since $\operatorname{Re}(m)>0$, the real part of the right hand side of (19) is also positive. Thus, any square root of the right hand side of (19) has a non-zero real part, and we can take $K$ as the square root of the right hand side of (19) with $\operatorname{Re}(K)>0$. The solutions $z_{1}, z_{2}$ of (19) are then given by

$$
z_{1}=K-\frac{3}{4}(\alpha+\beta), \quad z_{2}=-K-\frac{3}{4}(\alpha+\beta) .
$$


Since $\alpha, \beta$ are linearly independent over $\mathbb{R}$, we can write $K=\lambda_{1} \alpha+\lambda_{2} \beta$ for real numbers $\lambda_{1}, \lambda_{2}$. Substituting this into (19), we obtain

$$
\left(\lambda_{1} \alpha+\lambda_{2} \beta\right)^{2}=\frac{5}{4}\left(6 d-a-b+\frac{1}{4}(\alpha+\beta)^{2}\right) .
$$

Taking the real part of this equation, we obtain

$$
2 \lambda_{1} \lambda_{2} \sqrt{r_{1} r_{2}}=\frac{5}{4}\left(2 \operatorname{Re}(m)+\frac{1}{2} \sqrt{r_{1} r_{2}}\right)
$$

and hence $\operatorname{Re}(m)=\left(\frac{4}{5} \lambda_{1} \lambda_{2}-\frac{1}{4}\right) \sqrt{r_{1} r_{2}}$. Thus $\lambda_{1} \lambda_{2}>\frac{5}{16}$ since $\operatorname{Re}(m)>0$.

Now, $z_{1}, z_{2}$ can be expressed as

$$
z_{1}=\left(\lambda_{1}-\frac{3}{4}\right) \alpha+\left(\lambda_{2}-\frac{3}{4}\right) \beta, \quad z_{2}=-\left(\lambda_{1}+\frac{3}{4}\right) \alpha-\left(\lambda_{2}+\frac{3}{4}\right) \beta .
$$

If $z_{1}, z_{2}$ are both in $H_{\alpha, \beta} \cup W_{\alpha, \beta}^{0}$ or in $H_{\alpha, \beta} \cup W_{\alpha, \beta}^{1}$, then $\lambda_{1} \lambda_{2} \leq \frac{1}{16}$ (see Figure 16). Therefore, only one solution is in $W_{\alpha, \beta}^{0}$, and the other solution is in $W_{\alpha, \beta}^{1}$. Actually, $z_{1} \in W_{\alpha, \beta}^{0}$ from the following argument.

Suppose $z_{1} \notin W_{\alpha, \beta}^{0}$. Then $\lambda_{1}, \lambda_{2}$ must satisfy the following conditions.

$$
\lambda_{1}<\frac{3}{4}, \quad \lambda_{2}<\frac{3}{4}, \quad\left(\lambda_{1}-\frac{3}{4}\right)\left(\lambda_{2}-\frac{3}{4}\right) \geq \frac{1}{4} .
$$

Since

$$
\operatorname{Re}(K)=\left(\lambda_{1} \sqrt{r_{1}}+\lambda_{2} \sqrt{r_{2}}\right) / \sqrt{2}>0,
$$

$\lambda_{1}+\lambda_{2} R>0$ where $R=\sqrt{r_{2} / r_{1}}$. Thus, $\lambda_{1} \lambda_{2} \leq \frac{1}{16}$ by Lemma 9.5 (see Figure 16). But this is a contradiction, so $z_{1} \in W_{\alpha, \beta}^{0}$ and $z_{2} \in W_{\alpha, \beta}^{1}$.

Let $\chi(t)=\alpha(1-t)^{2}+z_{1} 2(1-t) t+\beta t^{2}$. Then $\operatorname{wind}\left(L_{\alpha, \beta} \ominus \chi\right)=0$.

(ii) $\operatorname{Re}(m)<0$ : From Lemma 8.4, $\Delta \theta_{r^{\prime}}=-\pi$, and if we set $s(0)=\alpha$, then $s(1)=-\beta$. So by Lemma 3.5, we need only consider $\chi(t)=\alpha(1-t)^{2}+$ $z 2(1-t) t-\beta t^{2}$ with $z$ satisfying

$$
\left(z+\frac{3}{4}(\alpha-\beta)\right)^{2}=\frac{5}{4}\left(6 d-a-b+\frac{1}{4}(\alpha-\beta)^{2}\right) .
$$

Consider the line segment $L_{\alpha,-\beta}=\alpha(1-t)-\beta t$ from $\alpha$ to $-\beta$. Since $\Delta \theta_{s}=\Delta \theta_{L_{\alpha,-\beta}}=-\pi / 2$,

$$
\operatorname{wind}\left(r^{\prime} \ominus \chi^{2}\right)=2 \operatorname{wind}(s \ominus \chi)=2 \operatorname{wind}\left(L_{\alpha,-\beta} \ominus \chi\right) .
$$

We need to show a solution $z$ of (20) exists so that $\operatorname{wind}\left(L_{\alpha,-\beta} \ominus \chi\right)=0$.

Since $\operatorname{Re}(m)<0$, the real part of the right hand side of $(\overline{20})$ is also negative. Thus, any square root of the right hand side of (20) has a nonzero imaginary part. Therefore, we can take $K$ as the square root of the right hand side of (20) so that $\operatorname{Im}(K)<0$.

Then two solutions $z_{1}$ and $z_{2}$ of (20) are given by

$$
z_{1}=K-\frac{3}{4}(\alpha-\beta), \quad z_{2}=-K-\frac{3}{4}(\alpha-\beta) .
$$



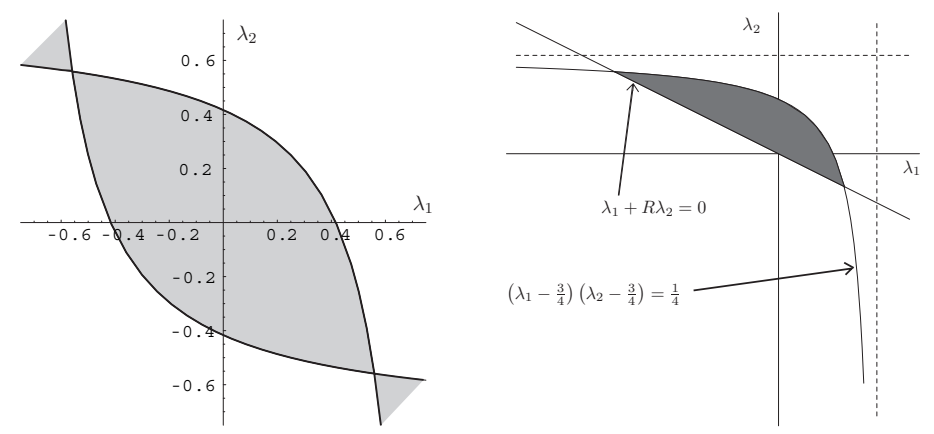

Figure 16: Illustrations for the proof of Theorem 8.5 .

Since $\alpha, \beta$ are linearly independent over $\mathbb{R}$, there exist real numbers $\lambda_{1}, \lambda_{2}$ such that $K=\lambda_{1} \alpha-\lambda_{2} \beta$. Substituting this into (20), we obtain

$$
\left(\lambda_{1} \alpha-\lambda_{2} \beta\right)^{2}=\frac{5}{4}\left(6 d-a-b+\frac{1}{4}(\alpha-\beta)^{2}\right) .
$$

Taking the real part of the above equation, we get

$$
-2 \lambda_{1} \lambda_{2} \sqrt{r_{1} r_{2}}=\frac{5}{4}\left(2 \operatorname{Re}(m)-\frac{1}{2} \sqrt{r_{1} r_{2}}\right)
$$

and hence $\operatorname{Re}(m)=\left(\frac{1}{4}-\frac{4}{5} \lambda_{1} \lambda_{2}\right) \sqrt{r_{1} r_{2}}$. Thus $\lambda_{1} \lambda_{2}>\frac{5}{16}$ since $\operatorname{Re}(m)<0$. Now $z_{1}, z_{2}$ can be expressed as

$z_{1}=\left(\lambda_{1}-\frac{3}{4}\right) \alpha+\left(\lambda_{2}-\frac{3}{4}\right)(-\beta), \quad z_{2}=-\left(\lambda_{1}+\frac{3}{4}\right) \alpha-\left(\lambda_{2}+\frac{3}{4}\right)(-\beta)$.

If both $z_{1}$ and $z_{2}$ are in $H_{\alpha,-\beta} \cup W_{\alpha,-\beta}^{0}$ or in $H_{\alpha,-\beta} \cup W_{\alpha,-\beta}^{1}$, then $\lambda_{1} \lambda_{2} \leq \frac{1}{16}$ (see Figure 16). Therefore, only one solution is in $W_{\alpha,-\beta}^{0}$, and the other solution is in $W_{\alpha,-\beta}^{1}$. Actually, $z_{1} \in W_{\alpha,-\beta}^{0}$ from the following argument. Suppose $z_{1} \notin W_{\alpha,-\beta}^{0}$. Then, $\lambda_{1}, \lambda_{2}$ must satisfy the following conditions.

$$
\lambda_{1}<\frac{3}{4}, \quad \lambda_{2}<\frac{3}{4}, \quad\left(\lambda_{1}-\frac{3}{4}\right)\left(\lambda_{2}-\frac{3}{4}\right) \geq \frac{1}{4} .
$$

Since

$$
\operatorname{Im}(K)=-\left(\lambda_{1} \sqrt{r_{1}}+\lambda_{2} \sqrt{r_{2}}\right) / \sqrt{2}<0
$$

$\lambda_{1}+\lambda_{2} R>0$, where $R=\sqrt{r_{2} / r_{1}}$. Thus, by Lemma $9.5, \lambda_{1} \lambda_{2} \leq \frac{1}{16}$ (see Figure 16). But this is a contradiction, so $z_{1} \in W_{\alpha,-\beta}^{0}$ and $z_{2} \in W_{\alpha,-\beta}^{1}$. Let $\chi(t)=\alpha(1-t)^{2}+z_{1} 2(1-t) t-\beta t^{2}$. Then $\operatorname{wind}\left(L_{\alpha,-\beta} \ominus \chi\right)=0$.

\section{Appendix 2: Proofs}

We present below some technical proofs for results used in the body of the paper. 
Lemma 9.1 Let $z_{1}, z_{2}$ be complex numbers. If $\mu \in W_{z_{1}, z_{2}}^{0}$ then $Q_{z_{1}, \mu, z_{2}}(t) \in$ $A_{z_{1}, z_{2}}$ for $t \in(0,1)$ and if $\mu \in W_{z_{1}, z_{2}}^{1}$ then $Q_{z_{1}, \mu, z_{2}}(t) \in B_{z_{1}, z_{2}}$ for $t \in(0,1)$.

Proof. For brevity we write $Q(t)$ for $Q_{z_{1}, \mu, z_{2}}(t)$. For $\mu=\lambda_{1} z_{1}+\lambda_{2} z_{2}$ we have

$$
Q(t)=\left((1-t)^{2}+2 \lambda_{1}(1-t) t\right) z_{1}+\left(t^{2}+2 \lambda_{2}(1-t) t\right) z_{2} .
$$

(i) Consider the first statement. Let $\mu \in W_{z_{1}, z_{2}}^{0}$. Then we only need check the following three cases.

(a) $\lambda_{1} \geq 0$ : Since $(1-t)^{2}+2 \lambda_{1}(1-t) t>0$ for $t \in(0,1), Q(t) \in A_{z_{1}, z_{2}}$ for all $t \in(0,1)$.

(b) $\lambda_{2} \geq 0$ : Since $t^{2}+2 \lambda_{2}(1-t) t>0$ for $t \in(0,1), Q(t) \in A_{z_{1}, z_{2}}$ for all $t \in(0,1)$.

(c) $\lambda_{1}<0, \lambda_{2}<0,4 \lambda_{1} \lambda_{2}<1$ : First note that

$$
0<\frac{-2 \lambda_{2}}{1-2 \lambda_{2}}<\frac{1}{1-2 \lambda_{1}}<1 .
$$

This is clear from the following inequality.

$$
\begin{aligned}
\frac{1}{1-2 \lambda_{1}}-\frac{-2 \lambda_{2}}{1-2 \lambda_{2}} & =\frac{\left(1-2 \lambda_{2}\right)+2 \lambda_{2}\left(1-2 \lambda_{1}\right)}{\left(1-2 \lambda_{1}\right)\left(1-2 \lambda_{2}\right)} \\
& =\frac{1-4 \lambda_{1} \lambda_{2}}{\left(1-2 \lambda_{1}\right)\left(1-2 \lambda_{2}\right)}>0 .
\end{aligned}
$$

If $0<t<1 /\left(1-2 \lambda_{1}\right)$, then

$$
\begin{aligned}
(1-t)^{2}+2 \lambda_{1}(1-t) t & =(1-t)\left(1-t+2 \lambda_{2} t\right) \\
& =(1-t)\left(1-\left(1-2 \lambda_{2}\right) t\right)>0 .
\end{aligned}
$$

If $-2 \lambda_{2} /\left(1-2 \lambda_{2}\right)<t<1$, then

$$
\begin{aligned}
t^{2}+2 \lambda_{2}(1-t) t & =t\left(t+2 \lambda_{2}(1-t)\right) \\
& =t\left(\left(1-2 \lambda_{2}\right) t+2 \lambda_{2}\right)>0 .
\end{aligned}
$$

Since $\left(0, \frac{1}{1-2 \lambda_{1}}\right) \cup\left(\frac{-2 \lambda_{2}}{1-2 \lambda_{2}}, 1\right)=(0,1), Q(t) \in A_{z_{1}, z_{2}}$ for $t \in(0,1)$.

(ii) For the second statement, let $\mu \in W_{z_{1}, z_{2}}^{1}$. Then $\lambda_{1}<0, \lambda_{2}<0,4 \lambda_{1} \lambda_{2}>1$. So, by similar calculations, we obtain the inequalities

$$
0<\frac{1}{1-2 \lambda_{1}}<\frac{-2 \lambda_{2}}{1-2 \lambda_{2}}<1 .
$$

If $1 /\left(1-2 \lambda_{1}\right)<t<1$, then

$(1-t)^{2}+2 \lambda_{1}(1-t) t=(1-t)\left(1-t+2 \lambda_{1} t\right)=(1-t)\left(1-\left(1-2 \lambda_{1}\right) t\right)<0$.

If $0<t<-2 \lambda_{2} /\left(1-2 \lambda_{2}\right)$, then

$$
t^{2}+2 \lambda_{2}(1-t) t=t\left(t+2 \lambda_{2}(1-t)\right)=t\left(\left(1-2 \lambda_{2}\right) t+2 \lambda_{2}\right)<0 .
$$

Since $\left(0, \frac{-2 \lambda_{2}}{1-2 \lambda_{2}}\right) \cup\left(\frac{1}{1-2 \lambda_{1}}, 1\right)=(0,1), Q(t) \in B_{z_{1}, z_{2}}$ for $t \in(0,1)$. 
For the following proofs, let $\log z$ denote the principal branch of $\log z$, such that $-\pi<\operatorname{Im}(\log z) \leq \pi$-i.e., $\log z=\log |z|+i \operatorname{Arg} z$.

Lemma 9.2 If $z_{1}, z_{2}$ are linearly independent over $\mathbb{R}$, then $L_{z_{1}, z_{2}}(t) \neq 0$ for $t \in[0,1]$. The angle variation $\Delta \theta_{L}$ of $L_{z_{1}, z_{2}}(t)$ for $t \in[0,1]$ is well defined, and $\Delta \theta_{L}=\operatorname{Arg}\left(z_{2} / z_{1}\right)$. So, clearly $\left|\Delta \theta_{L}\right|<\pi$.

Proof. Since $z_{1}, z_{2}$ are linearly independent over $\mathbb{R}$, and $1-t$ and $t$ cannot be both zero, it is clear that $L_{z_{1}, z_{2}}(t) \neq 0$. By taking a proper rotation $R$ with respect to the origin, we can set $R\left(z_{1}\right)=r_{1} e^{-i \theta}, R\left(z_{2}\right)=r_{2} e^{i \theta}$ for some positive $r_{1}, r_{2}$, and $\theta \in(-\pi / 2,0) \cup(0, \pi / 2)$. Since $\tilde{L}(t):=R(L(t))=R(a)(1-t)+R(b) t \in$ $D=\{x+i y \mid x>0, y \in \mathbb{R}\}$ and $\log z$ is analytic in the domain $D$,

$$
\begin{aligned}
\Delta \theta_{L} & =\Delta \theta_{\tilde{L}}=\operatorname{Im} \int_{\tilde{L}} \frac{1}{z} \mathrm{~d} z=\operatorname{Arg}(\tilde{L}(1))-\operatorname{Arg}(\tilde{L}(0)) \\
& =2 \theta=\operatorname{Arg}\left(R\left(z_{2}\right) / R\left(z_{1}\right)\right)=\operatorname{Arg}\left(z_{2} / z_{1}\right)
\end{aligned}
$$

Theorem 9.3 Let the complex numbers $z_{1}, z_{2}$ be linearly independent over $\mathbb{R}$. Then clearly the two open sets $A_{z_{1}, z_{2}}$ and $B_{z_{1}, z_{2}}$ do not contain 0 . If $\mu$ is not in $H_{z_{1}, z_{2}}$, the angle variation $\Delta \theta_{Q}$ of $Q(t)=z_{1}(1-t)^{2}+\mu 2 t(1-t)+z_{2} t^{2}, t \in[0,1]$ is well defined, and $\left|\Delta \theta_{Q}\right|<2 \pi$. Moreover, we have

(i) If $Q(t) \in A_{z_{1}, z_{2}}$ for all $t \in(0,1)$ then $\left|\Delta \theta_{Q \ominus L}\right|=\left|\Delta \theta_{Q}-\Delta \theta_{L}\right|=0$ and $\left|\Delta \theta_{Q}\right|<\pi$

(ii) If $Q(t) \in B_{z_{1}, z_{2}}$ for all $t \in(0,1)$ then $\left|\Delta \theta_{Q \ominus L}\right|=\left|\Delta \theta_{Q}-\Delta \theta_{L}\right|=2 \pi$ and $\pi<\left|\Delta \theta_{Q}\right|<2 \pi$.

Here $\Delta \theta_{L}$ is the angle variation of $L_{z_{1}, z_{2}}(t)$ for $t \in[0,1]$.

Proof. First note that since $z_{1}, z_{2}$ are linearly independent, $H_{z_{1}, z_{2}}, W_{z_{1}, z_{2}}^{0}$, and $W_{z_{1}, z_{2}}^{1}$ are mutually disjoint. If $\mu \notin H_{z_{1}, z_{2}}, \Delta \theta_{Q}$ is clearly well-defined by Theorem 3.2. Without loss of generality we can assume that $z_{1}=r_{1} e^{-i \theta}$, $z_{2}=r_{2} e^{i \theta}$ for some positive $r_{1}, r_{2}$, and $\theta \in(-\pi / 2,0) \cup(0, \pi / 2)$.

(i) Suppose $Q(t) \in A_{z_{1}, z_{2}}$ for $t \in(0,1)$. Since it is clear that $Q(0), Q(1) \in$ $A_{z_{1}, z_{2}}, Q(t) \in A_{z_{1}, z_{2}}$ for all $t \in[0,1]$. Since $\log z$ is analytic in $A_{z_{1}, z_{2}}$,

$$
\Delta \theta_{Q}=\operatorname{Im} \int_{Q} \frac{1}{z} \mathrm{~d} z=\operatorname{Arg}(Q(1))-\operatorname{Arg}(Q(0))=2 \theta=\Delta \theta_{L} .
$$

In this case, $\left|\Delta \theta_{Q}\right|=\left|\Delta \theta_{L}\right|<\pi$.

(ii) Now suppose that $Q(t) \in B_{z_{1}, z_{2}}$ for all $t \in(0,1)$. So $Q(t) \in D=\mathbb{C} \backslash\{x \in$ $\mathbb{R} \mid x \geq 0\}$ for all $t \in[0,1]$. Let $\log _{D} z$ be the branch of $\log z$ such that $\log _{D} z=\log |z|+i \operatorname{Arg}{ }_{D} z$, where $\operatorname{Arg}_{D} z$ is the argument of $z$ such that $0<\operatorname{Arg}{ }_{D} z \leq 2 \pi$. Then $\log _{D} z$ is analytic in $D$, and

$$
\operatorname{Arg}_{D}(Q(1))=\left\{\begin{array}{ll}
\theta, & \theta>0 \\
\theta+2 \pi, & \theta<0
\end{array} \quad, \quad \operatorname{Arg}_{D}(Q(0))= \begin{cases}-\theta+2 \pi, & \theta>0 \\
-\theta, & \theta<0\end{cases}\right.
$$


So,

$$
\Delta \theta_{Q}=\operatorname{Im} \int_{Q} \frac{1}{z} \mathrm{~d} z=\operatorname{Arg}_{D}(Q(1))-\operatorname{Arg}_{D}(Q(0))= \begin{cases}2 \theta-2 \pi & \text { if } \theta>0 ; \\ 2 \theta+2 \pi & \text { if } \theta<0 .\end{cases}
$$

Therefore, $\left|\Delta \theta_{Q}-\Delta \theta_{L}\right|=2 \pi$. Moreover, $\pi<\left|\Delta \theta_{Q}\right|<2 \pi$.

Proof of Theorem 3.3. This is a simple corollary of Lemma 9.1, Theorem 9.3 , and Theorem 3.2 .

Proof of Theorem 3.4. Clearly for $\mu \notin H_{z_{1}, z_{2}}, \Delta \theta_{\sqrt{Q}}$ is well defined since $\sqrt{Q}(t) \neq 0$ for all $t \in[0,1]$. Moreover, since $\sqrt{Q}(t)^{2}=Q(t)$, it is clear that $\Delta \theta_{\sqrt{Q}}=\Delta \theta_{Q} / 2$. Since $\sqrt{Q}(0)=L(0)$ and $\sqrt{Q}(1)=L(1)$ with $L(t) \in \mathbb{C} \backslash\{0\}$, $\left|\Delta \theta_{\sqrt{Q}}-\Delta \theta_{L}\right|=2 n \pi$ for some integer $n$. But, since

$$
\left|\Delta \theta_{\sqrt{Q}}-\Delta \theta_{L}\right| \leq\left|\Delta \theta_{\sqrt{Q}}\right|+\left|\Delta \theta_{L}\right|<\pi+\pi=2 \pi,
$$

$\left|\Delta \theta_{\sqrt{Q}}-\Delta \theta_{L}\right|=0$. Then, by Lemma 9.2 it is clear that

$$
\Delta \theta_{\sqrt{Q}}=\Delta \theta_{L}=\operatorname{Arg}(\sqrt{Q}(1) / \sqrt{Q}(0)) .
$$

Proof of Lemma 3.5. First, suppose $X(0)=Y(0)$ and $X(1)=Y(1)$. Then, since $X \ominus Y$ is a closed curve,

$$
\operatorname{wind}\left(X^{2} \ominus Y^{2}\right)=\frac{2}{2 \pi}\left(\Delta \theta_{X}-\Delta \theta_{Y}\right)=2 \operatorname{wind}(X \ominus Y) .
$$

Note that the winding number of a closed curve is integer. Now, suppose $X(0)=$ $Y(0)$ and $X(1)=-Y(1)$. Then,

$$
\operatorname{wind}\left(X^{2} \ominus Y^{2}\right)=\frac{2}{2 \pi}\left(\Delta \theta_{X}-\Delta \theta_{Y}\right) .
$$

But, since $X(1)=-Y(1)$,

$$
\begin{aligned}
\Delta \theta_{X}-\Delta \theta_{Y} & =\left(\operatorname{Arg} X(1)-\operatorname{Arg} X(0)+2 k_{1} \pi\right)-\left(\operatorname{Arg} Y(1)-\operatorname{Arg} Y(0)+2 k_{2} \pi\right) \\
& =\operatorname{Arg} X(1)-\operatorname{Arg} Y(1)+2\left(k_{1}-k_{2}\right) \pi=(2 n+1) \pi
\end{aligned}
$$

for some integer $k_{1}, k_{2}, n$. Thus, $\operatorname{wind}\left(X^{2} \ominus Y^{2}\right)=2 n+1$.

Proof of Lemma 4.2. From $s(t)^{2}=r^{\prime}(t), \chi(t)^{2}=\gamma^{\prime}(t)$, and Theorem 3.4,

$\operatorname{wind}\left(r^{\prime} \ominus \gamma^{\prime}\right)=\frac{1}{2 \pi}\left(\Delta \theta_{r^{\prime}}-\Delta \theta_{\gamma^{\prime}}\right)=\frac{1}{2 \pi}\left(2 \Delta \theta_{s}-2 \Delta \theta_{\chi}\right)=\frac{1}{2 \pi}\left(2 \Delta \theta_{L}-2 \Delta \theta_{\chi}\right)$, where $L(t)=s(0)(1-t)+s(1) t$. Thus, if $s(0)=\chi(0)$ and $s(1)=\chi(1)$ we have

$$
\operatorname{wind}\left(r^{\prime} \ominus \gamma^{\prime}\right)=2 \operatorname{wind}\left(L_{\chi(0), \chi(1)} \ominus \chi\right) .
$$


Since $\chi(t)$ is a quadratic Bézier curve, wind $\left(L_{\chi(0), \chi(1)} \ominus \chi\right)$ is 0 or \pm 1 from Theorem 3.3. So, wind $\left(r^{\prime} \ominus \gamma^{\prime}\right)$ is 0 or \pm 2 . Now, suppose $s(0)=\chi(0)$ and $s(1)=-\chi(1)$. In this case, clearly

$$
\Delta \theta_{L}-\Delta \theta_{\chi}=(2 n+1) \pi
$$

for some integer $n$. But, $\left|\Delta \theta_{L}\right|<\pi$ from Lemma 9.2, and $\left|\Delta \theta_{\chi}\right|<2 \pi$ from Theorem 9.3, so

$$
\left|\Delta \theta_{L}-\Delta \theta_{\chi}\right| \leq\left|\Delta \theta_{L}\right|+\left|\Delta \theta_{\chi}\right|<3 \pi \text {. }
$$

Therefore, $\left|\Delta \theta_{L}-\Delta \theta_{\chi}\right|=1$ and $\operatorname{wind}\left(r^{\prime} \ominus \gamma^{\prime}\right)= \pm 1$.

Proof of Lemma 4.1. First note that the middle control point of $r^{\prime}(t)$ is $m$. Thus, by Theorem 9.3, $m \in W_{a, b}^{0}$ implies that $\left|\Delta \theta_{r^{\prime}}\right|<\pi$, and $m \in W_{a, b}^{1}$ implies that $\pi<\left|\Delta \theta_{r^{\prime}}\right|<2 \pi$. Since $\Delta \theta_{s}=\Delta \theta_{r^{\prime}} / 2,\left|\Delta \theta_{s}\right|<\pi / 2$ when $m \in W_{a, b}^{0}$, and $\pi / 2<\left|\Delta \theta_{s}\right|<\pi$ when $m \in W_{a, b}^{1}$. We now calculate $\Delta \theta_{s}$ in terms of $\alpha, \beta$. If $\theta(t)$ is a continuous function of argument of $s(t)$, then

$$
\begin{aligned}
\Delta \theta_{s} & =\theta(1)-\theta(0)=\left(\operatorname{Arg} s(1)+2 n_{1} \pi\right)-\left(\operatorname{Arg} s(0)+2 n_{0} \pi\right) \\
& =\operatorname{Arg} s(1)-\operatorname{Arg} s(0)+2\left(n_{1}-n_{0}\right) \pi=\operatorname{Arg}(s(1) / \alpha)+2 k \pi
\end{aligned}
$$

for some integers $n_{1}, n_{0}, k$. However, since $|\operatorname{Arg}(s(1) / \alpha)|<\pi$ and $\left|\Delta \theta_{s}\right|<\pi, k$ must be 0 . Therefore, $\Delta \theta_{s}=\operatorname{Arg}(s(1) / \alpha)$. Now note that $|\operatorname{Arg}(\beta / \alpha)|<\pi / 2$ and $\pi / 2<|\operatorname{Arg}(-\beta / \alpha)|<\pi$. So, if $m \in W_{a, b}^{0}$, then $|\operatorname{Arg}(s(1) / \alpha)|=\left|\Delta \theta_{s}\right|<$ $\pi / 2$, thus $s(1)=\beta$. On the other hand, if $m \in W_{a, b}^{1}$, then $|\operatorname{Arg}(s(1) / \alpha)|=$ $\left|\Delta \theta_{s}\right|>\pi / 2$, thus $s(1)=-\beta$.

Suppose $a, b$ are in the form of standard configuration, i.e., $a=r_{1} e^{-i \theta}, b=$ $r_{2} e^{i \theta}, \alpha=\sqrt{r_{1}} e^{-i \theta / 2}, \beta=\sqrt{r_{2}} e^{i \theta / 2}$ for positive $r_{1}, r_{2}$ and $0<|\theta|<\pi / 2$.

Proof of Theorem 4.17. First we need the following lemma.

Lemma 9.4 For $d \in \mathbb{C}$, let $K$ be given by $K^{2}=\frac{5}{4}\left(6 d-a-b+\frac{1}{4}(\alpha+\beta)^{2}\right)$. Then if $m=3 d-a-b \in W_{a, b}^{0} \cup H_{a, b}$, we have $\operatorname{Re}(K) \neq 0$.

Proof of Lemma 9.4. Suppose $\operatorname{Re}(K)=0$. Then, $K^{2} \leq 0$ and

$$
m=\frac{2}{5} K^{2}-\frac{1}{2}(a+b)-\frac{1}{8}(\alpha+\beta)^{2}=\frac{2}{5} K^{2}-\frac{5}{8}(a+b)-\frac{1}{4} \alpha \beta=-\frac{5}{8}(a+b)-\epsilon
$$

for some positive $\epsilon$. Since

$$
\left(a / r_{1}\right)+\left(b / r_{2}\right)=2 \cos \theta
$$

$m$ can be rewritten as

$$
m=-\left(\frac{5}{8}+\frac{1}{2} \epsilon / r_{1} \cos \theta\right) a-\left(\frac{5}{8}+\frac{1}{2} \epsilon / r_{2} \cos \theta\right) b .
$$

Let $A, B$ be such that

$$
A=-\frac{5}{8}-\frac{\epsilon}{2 r_{1} \cos \theta}, \quad B=-\frac{5}{8}-\frac{\epsilon}{2 r_{2} \cos \theta} .
$$


Since $A<-\frac{5}{8}, B<-\frac{5}{8}, A B>\frac{1}{4}$. This means that $m \in W_{a, b}^{1}$ which contradicts $m \in W_{a, b}^{0} \cup H_{a, b}$. Therefore, $\operatorname{Re}(K) \neq 0$.

Now let $K$ be the root with positive real part, written as $K=\lambda_{1} \alpha+\lambda_{2} \beta$ for some real numbers $\lambda_{1}, \lambda_{2}$. Then

$$
\operatorname{Re}\left(\lambda_{1} \alpha+\lambda_{2} \beta\right)=\cos \frac{1}{2} \theta\left(\lambda_{1} \sqrt{r_{1}}+\lambda_{2} \sqrt{r_{2}}\right) .
$$

So $\lambda_{1} \sqrt{r_{1}}+\lambda_{2} \sqrt{r_{2}}$ is positive. Set $R=\sqrt{r_{2} / r_{1}}$. Then, $\lambda_{1}+\lambda_{2} R>0$. Since

$$
\left(\lambda_{1} \alpha+\lambda_{2} \beta\right)^{2}=\frac{5}{4}\left(6 d-a-b+\frac{1}{4}(\alpha+\beta)^{2}\right),
$$

$m=A a+B b$, where

$$
A=\frac{2}{5} \lambda_{1}^{2}-\frac{5}{8}+\left(\frac{4}{5} \lambda_{1} \lambda_{2}-\frac{1}{4}\right) \frac{R}{2 \cos \theta}, \quad B=\frac{2}{5} \lambda_{2}^{2}-\frac{5}{8}+\left(\frac{4}{5} \lambda_{1} \lambda_{2}-\frac{1}{4}\right) \frac{1}{2 \cos \theta R} .
$$

Suppose $z=\left(\lambda_{1}-\frac{3}{4}\right) \alpha+\left(\lambda_{2}-\frac{3}{4}\right) \beta \notin W_{\alpha, \beta}^{0}$. Then, $\lambda_{1}, \lambda_{2}$ satisfy

$$
\lambda_{1}<\frac{3}{4}, \quad \lambda_{2}<\frac{3}{4}, \quad\left(\lambda_{1}-\frac{3}{4}\right)\left(\lambda_{2}-\frac{3}{4}\right) \geq \frac{1}{4} .
$$

Thus, by Lemma 9.6, $A<0, B<0, A B>\frac{1}{4}$. Therefore, $m=A a+B b \in W_{a, b}^{1}$. This contradicts the hypothesis, so $z \in W_{\alpha, \beta}^{0}$.

To prove Lemma 9.6, we need the following simple result (see Figure 16):

Lemma 9.5 Let $R$ be a positive real number. If $\lambda_{1}, \lambda_{2}$ satisfy the conditions

$$
\lambda_{1}<\frac{3}{4}, \quad \lambda_{2}<\frac{3}{4}, \quad\left(\lambda_{1}-\frac{3}{4}\right)\left(\lambda_{2}-\frac{3}{4}\right) \geq \frac{1}{4}, \quad \lambda_{1}+R \lambda_{2} \geq 0,
$$

then $\lambda_{1} \lambda_{2} \leq \frac{1}{16}$.

Lemma 9.6 $R>0$ and $0<C \leq 1$ are given. Let $A, B$ be

$$
A=\frac{2}{5} \lambda_{1}^{2}-\frac{5}{8}+\left(\frac{4}{5} \lambda_{1} \lambda_{2}-\frac{1}{4}\right) \frac{R}{2 C}, \quad B=\frac{2}{5} \lambda_{2}^{2}-\frac{5}{8}+\left(\frac{4}{5} \lambda_{1} \lambda_{2}-\frac{1}{4}\right) \frac{1}{2 C R} .
$$

If $\lambda_{1}, \lambda_{2}$ satisfy the following conditions

$$
\lambda_{1}<\frac{3}{4}, \quad \lambda_{2}<\frac{3}{4}, \quad\left(\lambda_{1}-\frac{3}{4}\right)\left(\lambda_{2}-\frac{3}{4}\right) \geq \frac{1}{4}, \quad \lambda_{1}+R \lambda_{2} \geq 0,
$$

then $A<0, B<0$, and $A B>\frac{1}{4}$.

Proof of Lemma 9.6. Since $\lambda_{1}+R \lambda_{2} \geq 0, \lambda_{1} \geq 0$ or $\lambda_{2} \geq 0$.

(i) $\lambda_{1} \geq 0, \lambda_{2} \geq 0$ : First note that $\lambda_{1} \lambda_{2} \leq \frac{1}{16}$ from Lemma 9.5. Since $0 \leq \lambda_{1}^{2}<\left(\frac{3}{4}\right)^{2}, 0 \leq \lambda_{2}^{2}<\left(\frac{3}{4}\right)^{2}$, and $\lambda_{1} \lambda_{2} \leq \frac{1}{16}$,

$$
A<-\frac{4}{10}-\frac{R}{10 C}, \quad B<-\frac{4}{10}-\frac{1}{10 C R} .
$$

Therefore,

$$
A B>\frac{1}{100}\left(16+\frac{4 R}{C}+\frac{4}{C R}+\frac{1}{C^{2}}\right) \geq \frac{1}{100}\left(16+\frac{8}{C}+\frac{1}{C^{2}}\right) \geq \frac{1}{4} .
$$


(ii) $\lambda_{1} \geq 0, \lambda_{2}<0$ : Since $0 \leq \lambda_{1}^{2}<\left(\frac{3}{4}\right)^{2}, \lambda_{1} \lambda_{2} \leq 0$,

$$
A<\frac{2}{5} \lambda_{1}^{2}-\frac{5}{8}<-\frac{4}{10} .
$$

Therefore $A B>\frac{1}{4}$, since

$$
\begin{aligned}
B & =\frac{2}{5 R} \lambda_{2}\left(R \lambda_{2}+\frac{1}{C} \lambda_{1}\right)-\frac{5}{8}-\frac{1}{8 C R} \\
& <\frac{2}{5 R} \lambda_{2}\left(R \lambda_{2}+\lambda_{1}+\left(\frac{1}{C}-1\right) \lambda_{1}\right)-\frac{5}{8} \leq-\frac{5}{8} .
\end{aligned}
$$

(iii) $\lambda_{1}<0, \lambda_{2} \geq 0$ : Since $0 \leq \lambda_{2}^{2}<\left(\frac{3}{4}\right)^{2}, \lambda_{1} \lambda_{2} \leq 0$,

$$
B<\frac{2}{5} \lambda_{2}^{2}-\frac{5}{8}<-\frac{4}{10} .
$$

Therefore $A B>\frac{1}{4}$, since

$$
\begin{aligned}
A & =\frac{2}{5} \lambda_{1}\left(\lambda_{1}+\frac{R}{C} \lambda_{2}\right)-\frac{5}{8}-\frac{R}{8 C} \\
& <\frac{2}{5} \lambda_{1}\left(\lambda_{1}+R \lambda_{2}+\left(\frac{1}{C}-1\right) R \lambda_{2}\right)-\frac{5}{8} \leq-\frac{5}{8} .
\end{aligned}
$$

Proof of Theorem 4.18. First we need the following lemma.

Lemma 9.7 For $d \in \mathbb{C}$, let $K$ be given by $K^{2}=\frac{5}{4}\left(6 d-a-b+\frac{1}{4}(\alpha-\beta)^{2}\right)$. Then if $m=3 d-a-b \in W_{a, b}^{1} \cup H_{a, b}$, we have $\operatorname{Im}(K) \neq 0$.

Proof of Lemma 9.7. Suppose $\operatorname{Im}(K)=0$. Then, $K^{2} \geq 0$ and

$$
m=\frac{2}{5} K^{2}-\frac{1}{2}(a+b)-\frac{1}{8}(\alpha-\beta)^{2}=\frac{2}{5} K^{2}-\frac{5}{8}(a+b)+\frac{1}{4} \alpha \beta=-\frac{5}{8}(a+b)+\epsilon
$$

for some positive $\epsilon$, where $\epsilon \geq \frac{1}{4} \sqrt{r_{1} r_{2}}$. Since

$$
\left(a / r_{1}\right)+\left(b / r_{2}\right)=2 \cos \theta,
$$

$m$ can be rewritten as

$$
m=\left(\frac{\epsilon}{2 r_{1} \cos \theta}-\frac{5}{8}\right) a+\left(\frac{\epsilon}{2 r_{2} \cos \theta}-\frac{5}{8}\right) b .
$$

Let $A, B$ be such that

$$
A=-\frac{5}{8}+\frac{\epsilon}{2 r_{1} \cos \theta}, \quad B=-\frac{5}{8}+\frac{\epsilon}{2 r_{2} \cos \theta} .
$$

Then,

$$
A>-\frac{5}{8}+\frac{1}{8} \sqrt{r_{2} / r_{1}}, \quad B>-\frac{5}{8}+\frac{1}{8} \sqrt{r_{1} / r_{2}} .
$$

Since, by the assumption, $m \in W_{a, b}^{1} \cup H_{a, b}, A$ and $B$ must be negative. So,

$$
\begin{aligned}
A B & <\frac{1}{64}\left(-5+\sqrt{r_{2} / r_{1}}\right)\left(-5+\sqrt{r_{1} / r_{2}}\right) \\
& =\frac{1}{64}\left(25-5 \sqrt{r_{2} / r_{1}}-5 \sqrt{r_{1} / r_{2}}+1\right) \leq \frac{1}{64}(25-10+1)=\frac{1}{4} .
\end{aligned}
$$


This is a contradiction to $m \in W_{a, b}^{1} \cup H_{a, b}$. So, $\operatorname{Im}(K) \neq 0$.

Now, let $K$ be the square root with $\sin \frac{1}{2} \theta \cdot \operatorname{Im} K<0$. We write $K=\lambda_{1} \alpha-\lambda_{2} \beta$ for some real numbers $\lambda_{1}, \lambda_{2}$. Then,

$$
\operatorname{Im}\left(\lambda_{1} \alpha-\lambda_{2} \beta\right)=-\sin \frac{1}{2} \theta\left(\lambda_{1} \sqrt{r_{1}}+\lambda_{2} \sqrt{r_{2}}\right) .
$$

So $\lambda_{1} \sqrt{r_{1}}+\lambda_{2} \sqrt{r_{2}}$ is positive. Set $R=\sqrt{r_{2} / r_{1}}$. Then, $\lambda_{1}+\lambda_{2} R>0$. Since

$$
\left(\lambda_{1} \alpha-\lambda_{2} \beta\right)^{2}=\frac{5}{4}\left(6 d-a-b+\frac{1}{4}(\alpha-\beta)^{2}\right),
$$

$m=A a+B b$, where

$$
A=\frac{2}{5} \lambda_{1}^{2}-\frac{5}{8}-\left(\frac{4}{5} \lambda_{1} \lambda_{2}-\frac{1}{4}\right) \frac{R}{2 \cos \theta}, \quad B=\frac{2}{5} \lambda_{2}^{2}-\frac{5}{8}-\left(\frac{4}{5} \lambda_{1} \lambda_{2}-\frac{1}{4}\right) \frac{1}{2 \cos \theta R} .
$$

Then, from the hypothesis $m \in W_{a, b}^{1} \cup H_{a, b}$, it is clear that $A<0$ and $B<0$. Suppose $z=\left(\lambda_{1}-\frac{3}{4}\right) \alpha-\left(\lambda_{2}-\frac{3}{4}\right) \beta \notin W_{\alpha,-\beta}^{0}$. Then $\lambda_{1}, \lambda_{2}$ satisfy the conditions

$$
\lambda_{1}<\frac{3}{4}, \quad \lambda_{2}<\frac{3}{4}, \quad\left(\lambda_{1}-\frac{3}{4}\right)\left(\lambda_{2}-\frac{3}{4}\right) \geq \frac{1}{4} .
$$

Thus, by Lemma 9.8, $A B<\frac{1}{4}$. This contradicts the hypothesis $m \in W_{a, b}^{1} \cup H_{a, b}$ so $z \in W_{\alpha,-\beta}^{0}$.

Lemma 9.8 Let $R>0$ and $0<C \leq 1$ and let $A, B$ be given by

$$
A=\frac{2}{5} \lambda_{1}^{2}-\frac{5}{8}-\left(\frac{4}{5} \lambda_{1} \lambda_{2}-\frac{1}{4}\right) \frac{R}{2 C}, \quad B=\frac{2}{5} \lambda_{2}^{2}-\frac{5}{8}-\left(\frac{4}{5} \lambda_{1} \lambda_{2}-\frac{1}{4}\right) \frac{1}{2 C R} .
$$

Also, let $\lambda_{1}, \lambda_{2}$ satisfy the following conditions

$$
\lambda_{1}<\frac{3}{4}, \quad \lambda_{2}<\frac{3}{4}, \quad\left(\lambda_{1}-\frac{3}{4}\right)\left(\lambda_{2}-\frac{3}{4}\right) \geq \frac{1}{4}, \quad \lambda_{1}+R \lambda_{2} \geq 0 .
$$

Then if $A<0, B<0$, we have $A B<\frac{1}{4}$.

Proof of Lemma 9.8 Let $M_{1}, M_{2}, N$ be given by

$$
M_{1}=\frac{5}{8}-\frac{2}{5} \lambda_{1}^{2}, \quad M_{2}=\frac{5}{8}-\frac{2}{5} \lambda_{2}^{2}, \quad N=\frac{1}{8}-\frac{2}{5} \lambda_{1} \lambda_{2} .
$$

From the conditions on $\lambda_{1}, \lambda_{2}$, we can easily deduce that $\lambda_{1} \lambda_{2} \leq \frac{1}{16}$. So $N$ is positive. Therefore, we get the following inequalities

$$
A>-M_{1}+N R, \quad B>-M_{2}+N / R .
$$

Since, by the assumption, $A<0$ and $B<0$,

$$
M_{1}>N R, \quad M_{2}>N / R .
$$

Therefore, $M_{1}$ and $M_{2}$ are positive, and $M_{1} M_{2}>N^{2}$. Moreover,

$$
\begin{aligned}
A B & <\left(-M_{1}+N R\right)\left(-M_{2}+N / R\right)=M_{1} M_{2}-N\left(M_{2} R+M_{1} / R\right)+N^{2} \\
& \leq M_{1} M_{2}-2 N \sqrt{M_{1} M_{2}}+N^{2}=\left(\sqrt{M_{1} M_{2}}-N\right)^{2} .
\end{aligned}
$$

Therefore $A B<\frac{1}{4}$, since

$$
0<\sqrt{M_{1} M_{2}}-N \leq \frac{1}{2}\left(M_{1}+M_{2}\right)-N=\frac{1}{2}-\frac{1}{5}\left(\lambda_{1}-\lambda_{2}\right)^{2} .
$$




\section{References}

[1] Rachid Ait Haddou and Luc Biard. $G^{2}$ approximation of an offset curve by Tschirnhausen quartics. In Mathematical methods for curves and surfaces (Ulvik, 1994), pages 1-10. Vanderbilt Univ. Press, Nashville, TN, 1995.

[2] Gudrun Albrecht and Rida T. Farouki. Construction of $C^{2}$ Pythagoreanhodograph interpolating splines by the homotopy method. Adv. Comput. Math., 5(4):417-442, 1996.

[3] Hyeong In Choi, Doo Seok Lee, and Hwan Pyo Moon. Clifford algebra, spin representation, and rational parameterization of curves and surfaces. Adv. Comput. Math., 17(1-2):5-48, 2002.

[4] Manjunathaiah J. Nicholas D.-Yuan G.-F. Jee S. Farouki, R. T. Variablefeedrate cnc interpolators for constant material removal rates along pythagorean-hodograph curves. Computer-Aided Design, 30:631-640, 1998.

[5] Manjunathaiah J. Yuan G.-F. Farouki, R. T. G codes for the specification of pythagorean-hodograph tool paths and associated feedrate functions on open-architecture cnc machines. Internat. J. Machine Tools and Manufacture, 39:123-142, 1999.

[6] R. T. Farouki and C. A. Neff. Hermite interpolation by Pythagorean hodograph quintics. Math. Comp., 64(212):1589-1609, 1995.

[7] R. T. Farouki and T. Sakkalis. Pythagorean hodographs. IBM J. Res. Develop., 34(5):736-752, 1990.

[8] Rida T. Farouki. Pythagorean-hodograph curves in practical use. In Geometry processing for design and manufacturing, pages 3-33. SIAM, Philadelphia, PA, 1992.

[9] Rida T. Farouki. The conformal map $z \rightarrow z^{2}$ of the hodograph plane. Comput. Aided Geom. Design, 11(4):363-390, 1994.

[10] Rida T. Farouki. The elastic bending energy of Pythagorean-hodograph curves. Comput. Aided Geom. Design, 13(3):227-241, 1996.

[11] Sagar Shah Farouki, R. T. Real-time cnc interpolators for pythagoreanhodograph curves, computer aided geometric design. Computer-Aided Design, 13(7):583-600, 1996.

[12] B. Jüttler. Hermite interpolation by Pythagorean hodograph curves of degree seven. Math. Comp., 70(235):1089-1111 (electronic), 2001.

[13] Hwan Pyo Moon. Computing rational offsets via media axis transform using polynomial speed curves in $\mathbb{R}^{2,1}$. In Geometric Modeling and Processing '98, pages 187-203. Bookplus Press, 1998. 
[14] Hwan Pyo Moon. Minkowski Pythagorean hodographs. Comput. Aided Geom. Design, 16(8):739-753, 1999.

[15] Hwan Pyo Moon, Rida T. Farouki, and Hyeong In Choi. Construction and shape analysis of PH quintic Hermite interpolants. Comput. Aided Geom. Design, 18(2):93-115, 2001.

[16] Helmut Pottmann. Curve design with rational Pythagorean-hodograph curves. Adv. Comput. Math., 3(1-2):147-170, 1995.

[17] Helmut Pottmann. Rational curves and surfaces with rational offsets. Comput. Aided Geom. Design, 12(2):175-192, 1995. 